\title{
Ammonia observations towards the Aquila Rift cloud complex
}

\author{
Kadirya Tursun ${ }^{1,2}$, Jarken Esimbek ${ }^{1,3}$, Christian Henkel ${ }^{4,5,1}$, Xindi Tang ${ }^{1,3,4}$, Gang $\mathrm{Wu}^{1,3}$, Dalei $\mathrm{Li}^{1,3}$, \\ Jianjun Zhou ${ }^{1,3}$, Yuxin He ${ }^{1,3}$, Toktarkhan Komesh ${ }^{1,2,6}$, and Serikbek Sailanbek ${ }^{1,2,6}$ \\ ${ }^{1}$ Xinjiang Astronomical Observatory, Chinese Academy of Sciences, 830011 Urumqi, PR China \\ e-mail: jarken@xao.ac.cn; tangxindi@xao.ac.cn \\ ${ }^{2}$ University of Chinese Academy of Sciences, 100080 Beijing, PR China \\ ${ }^{3}$ Key Laboratory of Radio Astronomy, Chinese Academy of Sciences, 830011 Urumqi, PR China \\ ${ }^{4}$ Max-Planck-Institut für Radioastronomie, Auf dem Hügel 69, 53121 Bonn, Germany \\ e-mail: chenkel@mpifr-bonn.mpg.de \\ 5 Astronomy Department, King Abdulaziz University, PO Box 80203, 21589 Jeddah, Saudi Arabia \\ ${ }^{6}$ Department of Solid State Physics and Nonlinear Physics, Faculty of Physics and Technology, AL-Farabi Kazakh National \\ University, 050040 Almaty, Kazakhstan
}

Received 4 February 2020 / Accepted 23 September 2020

\begin{abstract}
We surveyed the Aquila Rift complex including the Serpens South and W 40 regions in the $\mathrm{NH}_{3}(1,1)$ and $(2,2)$ transitions making use of the Nanshan $26-\mathrm{m}$ telescope. Our observations cover an area of $\sim 1.5^{\circ} \times 2.2^{\circ}(11.4 \mathrm{pc} \times 16.7 \mathrm{pc})$. The kinetic temperatures of the dense gas in the Aquila Rift complex obtained from $\mathrm{NH}_{3}(2,2) /(1,1)$ ratios range from 8.9 to $35.0 \mathrm{~K}$ with an average of $15.3 \pm 6.1 \mathrm{~K}$ (errors are standard deviations of the mean). Low gas temperatures are associated with Serpens South ranging from 8.9 to $16.8 \mathrm{~K}$ with an average of $12.3 \pm 1.7 \mathrm{~K}$, while dense gas in the $\mathrm{W} 40$ region shows higher temperatures ranging from 17.7 to $35.0 \mathrm{~K}$ with an average of $25.1 \pm 4.9 \mathrm{~K}$. A comparison of kinetic temperatures derived from para- $\mathrm{NH}_{3}(2,2) /(1,1)$ against $\mathrm{HiGal}$ dust temperatures indicates that the gas and dust temperatures are in agreement in the low-mass-star formation region of Serpens South. In the high-mass-star formation region $\mathrm{W} 40$, the measured gas kinetic temperatures are higher than those of the dust. The turbulent component of the velocity dispersion of $\mathrm{NH}_{3}(1,1)$ is found to be positively correlated with the gas kinetic temperature, which indicates that the dense gas may be heated by dissipation of turbulent energy. For the fractional total- $\mathrm{NH}_{3}$ (para+ortho) abundance obtained by a comparison with Herschel infrared continuum data representing dust emission, we find values from $0.1 \times 10^{-8}$ to $2.1 \times 10^{-7}$ with an average of $6.9( \pm 4.5) \times 10^{-8}$. Serpens South also shows a fractional total- $\mathrm{NH}_{3}$ (para+ortho) abundance ranging from $0.2 \times 10^{-8}$ to $2.1 \times 10^{-7}$ with an average of $8.6( \pm 3.8) \times 10^{-8}$. In W 40, values are lower, between 0.1 and $4.3 \times 10^{-8}$ with an average of $1.6( \pm 1.4) \times 10^{-8}$. Weak velocity gradients demonstrate that the rotational energy is a negligible fraction of the gravitational energy. In W 40, gas and dust temperatures are not strongly dependent on the projected distance to the recently formed massive stars. Overall, the morphology of the mapped region is ring-like, with strong emission at lower and weak emission at higher Galactic longitudes. However, the presence of a physical connection between the two parts remains questionable.
\end{abstract}

Key words. surveys - radio lines: ISM - ISM: molecules - ISM: kinematics and dynamics - stars: formation

\section{Introduction}

Systematic studies of dense molecular cores in regions of highmass-star formation (HMSF) are of great importance for our understanding of their physical and chemical properties. In comparison with low-mass star-forming regions, only a few, rather arbitrarily selected cores associated with HMSF regions have been investigated in some detail (Zinnecker \& Yorke 2007; Tan et al. 2014). There is a clear observational dichotomy between low-mass-star formation (LMSF) and HMSF. It is suggested that more than $70 \%$ of HMSF stars are formed in dense clusters embedded within giant molecular clouds (GMCs; Lada \& Lada 2003). For a better understanding of massive star formation, it is important to obtain the physical conditions of HMSF regions. High-mass stars form almost exclusively in GMCs while low-mass stars can form in dark clouds as well as in GMCs. High-mass stars are predominantly formed in clusters, while low-mass stars may form in smaller molecular complexes as for example in the Taurus molecular cloud or in isolation. The star formation efficiency is generally higher in HMSF regions (e.g., Myers et al. 1986; Lada \& Lada 2003). In HMSF regions, densities and temperatures tend to be higher, and spectral line widths are larger indicating a higher degree of turbulence. However, the causal relationship between the presence of young high-mass stars, molecular cloud characteristics, and details of the star formation process is not well established.

The star formation region in the Aquila Rift is located at a distance of $\sim 436 \pm 9.2$ pc (e.g., Ortiz-León et al. 2017, 2018) in areas extending up to Galactic latitudes of at least $+10^{\circ}$ and down to $-20^{\circ}$ (e.g., Dobashi et al. 2005). The Aquila Rift contains several active star-forming regions: Serpens Main, Serpens South, Serpens MWC297, and W 40. Here, we focus on that part of the Aquila Rift complex that harbors two known sites of star formation: Serpens South and W 40. Serpens South is a well-known site of star formation located in the western part of the Aquila Rift cloud complex and forms a young embedded stellar cluster (Gutermuth et al. 2008). It has a filamentary structure on the cusp of a burst of LMSF. W 40, located further to the east in equatorial coordinates, is a site of ongoing HMSF. It still contains dense molecular cores (Dobashi et al. 2005) and includes a blistered $\mathrm{H}$ II region, powered by a compact OB association that contains pre-main sequence stars (e.g., Zeilik \& Lada 1978; 
Smith et al. 1985; Vallee 1987; Kuhn et al. 2010; Rodríguez et al. 2010; Mallick et al. 2013).

Aquila is a unique region for studying the physical and chemical conditions of molecular clouds. A large number of molecular line observations have been performed, such as in $\mathrm{CO}$ (Nakamura et al. 2017; Su et al. 2019, 2020), in $\mathrm{NH}_{3}$ (Levshakov et al. 2013, 2014; Friesen et al. 2016) and in $\mathrm{H}_{2} \mathrm{CO}$ (Komesh et al. 2019). This was complemented by SCUBA-2 450 and $850 \mu \mathrm{m}$ observations (Rumble et al. 2016). A few years ago, the whole Aquila complex was extensively studied by the Herschel Gould Belt Survey ${ }^{1}$, yielding more than 500 detections of starless cores imaged in dust emission at 70-500 $\mu \mathrm{m}$ (Könyves et al. 2010). However, a systematic spectral survey of the dense gas in the region covering and comparing the emission of both Serpens South and the W 40 complex is still missing. A basic result of the $\mathrm{NH}_{3}$ studies of Levshakov et al. (2013, 2014), mostly carried out in position-switching mode, was that they were often finding unknown clouds at the OFF positions, providing the urgent need for a systematic survey of the entire region. Friesen et al. (2016) presented $\mathrm{NH}_{3}$ measurements of Serpens South $(\sim 4 \mathrm{pc} \times 4 \mathrm{pc})$ with the Green Bank Telescope (GBT). Here we present a complementary survey with lower angular resolution but a twelve-times-larger area encompassing many Aquila rift clouds with substantial visual extinction.

Ammonia $\left(\mathrm{NH}_{3}\right)$ is frequently used as the standard molecular cloud thermometer (e.g., Ho \& Townes 1983; Walmsley \& Ungerechts 1983; Danby et al. 1988); it starts to form at an early stage of prestellar core evolution and becomes brightest during the later stages (e.g., Suzuki et al. 1992). $\mathrm{NH}_{3}(1,1)$ and $(2,2)$, both belonging to the para-species of ammonia, have proven to form an excellent thermometer at $T_{\text {kin }}<40 \mathrm{~K}$ (Walmsley \& Ungerechts 1983). They can also serve as a good thermometer for higher temperatures after some modification of the rotational temperature but with a reduction in precision (Walmsley \& Ungerechts 1983; Danby et al. 1988; Tafalla et al. 2004). Moreover, the critical densities of $\mathrm{NH}_{3}(1,1)$ and $(2,2)$ are about $10^{3} \mathrm{~cm}^{-3}$ (Evans 1999; Shirley 2015), thus providing a proper tracer for dense regions.

In this paper we intend to provide novel ammonia maps covering the entire region of the Aquila Rift cloud complex and to reveal a complete distribution of the dense gas. The article is organized as follows: in Sect. 2 we introduce our observations and data reduction. Results are highlighted in Sect. 3. We discuss the variation of $\mathrm{NH}_{3}$ abundance and gas temperature in Sect. 4. Our main conclusions are summarized in Sect. 5.

\section{Observations and data reduction}

\section{1. $\mathrm{NH}_{3}$ observations}

From March 2017 to August 2018, we observed the $\mathrm{NH}_{3}(1,1)$ and $(2,2)$ lines with the Nanshan 26-m radio telescope. A 22.0 $24.2 \mathrm{GHz}$ dual polarization channel superheterodyne receiver was used. The main parameters of the Nanshan telescope and survey area are listed in Table 1 . The rest frequency was centered at $23.708 \mathrm{GHz}$ to observe $\mathrm{NH}_{3}(1,1)$ at $23.694 \mathrm{GHz}$ and $(2,2)$ at $23.723 \mathrm{GHz}$ simultaneously. To convert antenna temperatures $T_{A}^{*}$ into main beam brightness temperatures $T_{\mathrm{MB}}$, a beam efficiency of 0.59 was adopted. The observations were calibrated against periodically $(6 \mathrm{~s})$ injected signals from a noise diode. We observed IRAS 0033+636 $(\alpha=00: 36: 47.51, \delta=63: 29: 02.1$, J2000) repeatedly during observations (Schreyer et al. 1996),

1 http://www .herschel.fr/cea/gouldbelt/en/index.php
Table 1. Main parameters of the Nanshan telescope and survey area.

\begin{tabular}{ll}
\hline \hline Telescope & Nanshan 26-m antenna \\
Receiver & $K$-band receiver \\
Mode & On-The-Fly \\
Molecular lines & $\mathrm{NH}_{3}(1,1)$ and $(2,2)$ \\
Rest frequency & $23.708 \mathrm{GHz}$ \\
Bandwidth & $64 \mathrm{MHz}$ \\
Channel number & 8192 \\
Beam size & $\sim 2$ arcmin \\
Velocity resolution & $\sim 0.1 \mathrm{~km} \mathrm{~s}^{-1}$ \\
Main beam efficiency & $59 \%$ \\
Region & $28.2^{\circ}<l<29.7^{\circ}, 2.6^{\circ}<b<4.8^{\circ}$ \\
Survey area & $1.5^{\circ} \times 2.2^{\circ}$ \\
Serpens South & $28.5^{\circ}<l<29.1^{\circ}, 3.6^{\circ}<b<4.4^{\circ}$ \\
W 40 & $28.5^{\circ}<l<29.1^{\circ}, 3.2^{\circ}<b<3.6^{\circ}$ \\
\hline
\end{tabular}

adopting a main beam brightness temperature $T_{\mathrm{MB}}=4.5 \mathrm{~K}$. Systematic variations and dispersion of brightness temperatures are small (see Appendix A). The standard deviation of the mean is $\sim 10 \%$ (see Fig. A.1). To further check our calibration stability, we compared our $\mathrm{NH}_{3}$ data with previously taken $\mathrm{NH}_{3}$ data observed with the GBT in Fig. A.2 (Sokolov et al. 2017). The two data sets obtained from G035.39-0.33 are in good agreement.

The typical system temperature was $\sim 50 \mathrm{~K}\left(T_{A}^{*}\right.$ scale) at 23.708 GHz. The map was made using the on-the-fly (OTF) mode with a $6^{\prime} \times 6^{\prime}$ grid size and $30^{\prime \prime}$ sample step. All observations were obtained under excellent weather conditions and above an elevation of $20^{\circ}$. The mapped region was divided into six areas (see Fig. 1). The main parameters of each area are listed in Table A.1. The whole map covers a region of $\sim 1.5^{\circ} \times 2.2^{\circ}$ $(11.4 \mathrm{pc} \times 16.7 \mathrm{pc})$.

\subsection{Data reduction}

The CLASS and GREG packages of GILDAS ${ }^{2}$, and also python plot packages matplotlib (Hunter 2007) and APLpy ${ }^{3}$ were used for all the data reduction. The spectra were resampled in steps of $\sim 1^{\prime}$. To enhance signal-to-noise ratios $(\mathrm{S} / \mathrm{Ns})$ in individual channels, we smoothed in many but not all cases (see Sect. 3.1) contiguous channels to a velocity resolution of $\sim 0.2 \mathrm{~km} \mathrm{~s}^{-1}$. A typical rms noise level $(1 \sigma)$ is $\sim 0.03-0.05 \mathrm{~K}\left(T_{\mathrm{MB}}\right.$ scale) for a channel of $\sim 0.2 \mathrm{~km} \mathrm{~s}^{-1}$ in width (see Table A.1). With respect to $\mathrm{NH}_{3}$, we chose two fitting methods: "GAUSS" fit and $\mathrm{NH}_{3}(1,1)$ fit. In order to convert hyperfine blended line widths to intrinsic line widths in the $\mathrm{NH}_{3}$ inversion spectrum (e.g., Barranco \& Goodman 1998), we also fitted the averaged spectra using the GILDAS built-in " $\mathrm{NH}_{3}(1,1)$ " fitting method which can fit all 18 hyperfine components simultaneously. From the $\mathrm{NH}_{3}(1,1)$ fit we can obtain integrated intensity, line center velocity, intrinsic line widths of individual hyperfine structure (hfs) components, and optical depth. Main beam brightness temperatures $T_{\mathrm{MB}}$ are obtained from GAUSS fit. These fitting methods were also used in previous works, such as by Wienen et al. (2012) and by $\mathrm{Wu}$ et al. (2018). Examples for reduced and calibrated spectra of $\mathrm{NH}_{3}(1,1)$ and $(2,2)$ inversion lines are given in Fig. 2.

Because the hyperfine satellite lines of the $\mathrm{NH}_{3}(2,2)$ transition are mostly weak, $\mathrm{NH}_{3}(2,2)$ optical depths are not determined. A single Gaussian profile was fitted to the main group of

\footnotetext{
2 http://wWw.iram.fr/IRAMFR/GILDAS/

3 http://aplpy.github.com
} 


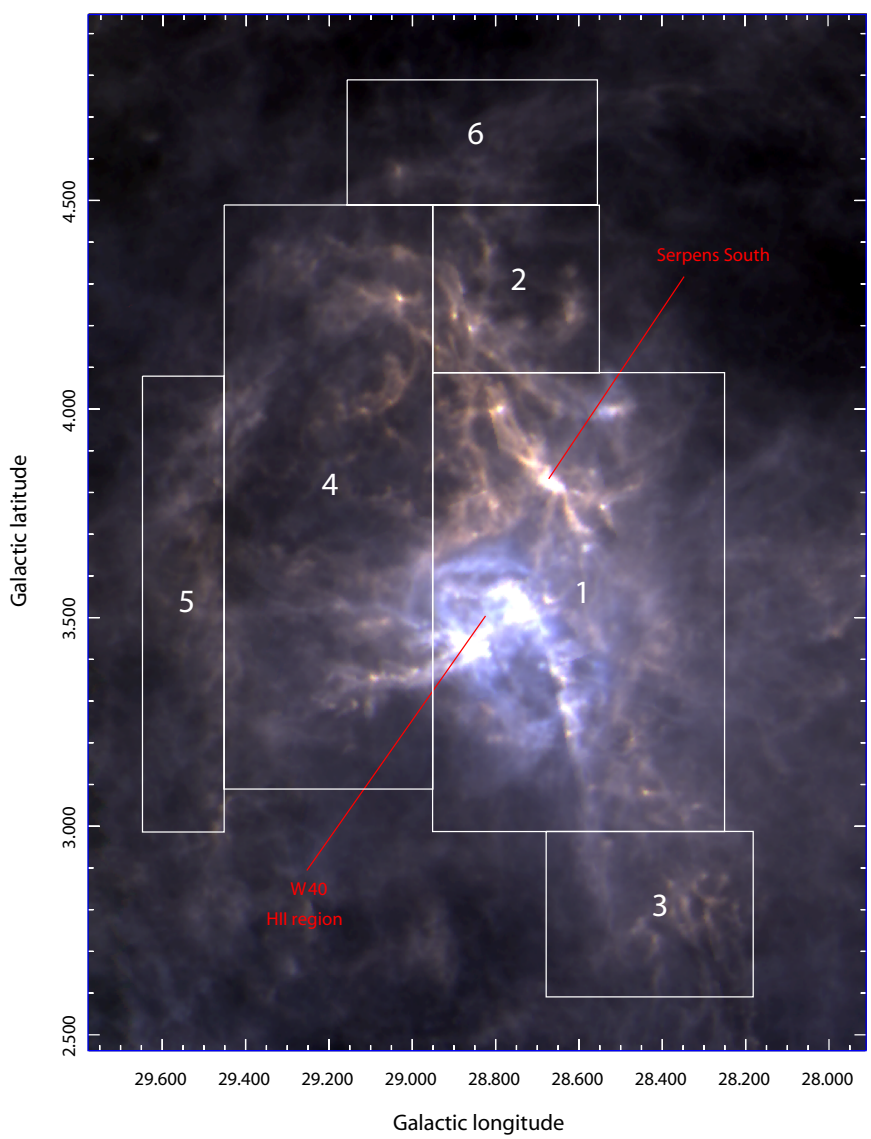

Fig. 1. Color image of the Aquila Rift (red for $500 \mu \mathrm{m}$, green for $350 \mu \mathrm{m}$, and blue for $250 \mu \mathrm{m}$, all derived from Herschel data; Bontemps et al. 2010; Könyves et al. 2010). The regions subsequently observed in $\mathrm{NH}_{3}$ are marked by six boxes (see Table A.1).

$\mathrm{NH}_{3}(2,2)$ hyperfine components. Physical parameters of dense gas such as rotational temperature $\left(T_{\text {rot }}\right)$, kinetic temperature $\left(T_{\text {kin }}\right)$, and para- $\mathrm{NH}_{3}$ column density $\left(N_{\mathrm{NH}_{3}}\right)$ were derived using the method described by Ho \& Townes (1983) and Ungerechts et al. (1986) (see Sects. 3.2 and 3.3).

\section{Results}

\section{1. $\mathrm{NH}_{3}$ distribution}

$\mathrm{NH}_{3}(1,1)$ and $(2,2)$ velocity-integrated intensity maps of the main groups of hyperfine components are presented in Fig. 3. Intensities were integrated over the Local Standard of Rest velocity $\left(V_{\mathrm{LSR}}\right)$ range of $4-10 \mathrm{~km} \mathrm{~s}^{-1} \cdot \mathrm{NH}_{3}(1,1)$ shows an extended distribution and clearly traces the dense molecular structure including Serpens South and W 40. $\mathrm{NH}_{3}(2,2)$ is only detected in the densest regions of Serpens South and W 40, and shows a much less extended distribution.

What we find in Fig. 3 is an Aquila Rift morphology that resembles the Herschel color infrared image shown in Fig. 1 in many aspects, but also shows clear differences (see also Fig. 1 of Könyves et al. 2010 for an $\mathrm{H}_{2}$ column density map of the region, based on Herschel data). In the Serpens South region, there is a dominant ridge of strong $\mathrm{NH}_{3}(1,1)$ emission from the southwest to the northeast (position angle $\sim 30^{\circ}$ in Galactic coordinates) containing several cores with a total length of about $15^{\prime}$. This is also seen in the color Herschel infrared image, but the latter is

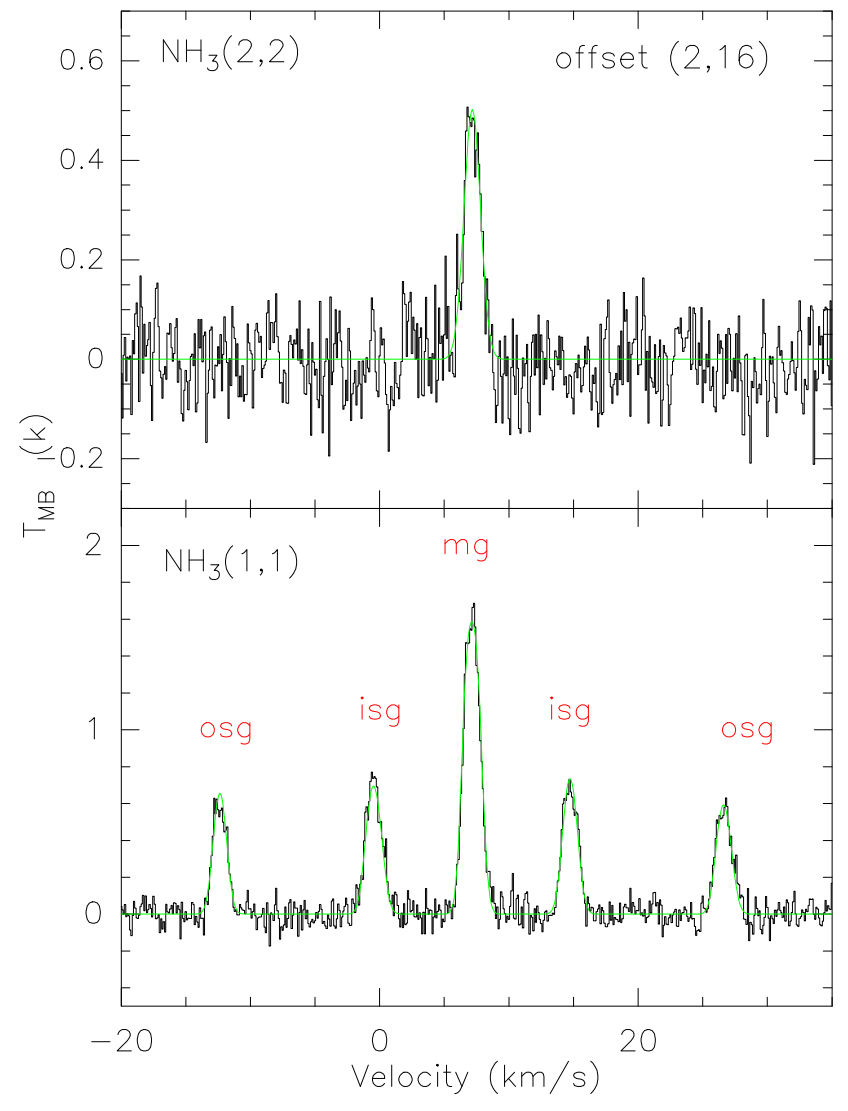

Fig. 2. $\mathrm{NH}_{3}(1,1)$ and $(2,2)$ spectra at offset $(\Delta l, \Delta b)=\left(2^{\prime}, 16^{\prime}\right)$ with respect to the reference position $\left(l=28.59^{\circ}, b=3.55^{\circ}\right)$. In each panel, the black solid line represents the observed spectrum, the green solid line indicates the $\mathrm{NH}_{3}(1,1)$ fitting (lower panel) and Gaussian fitting (upper panel) of the $\mathrm{NH}_{3}(2,2)$ line (see Sect. 2.2). The groups of hyperfine components "mg", "isg", and "osg" represent the main, inner-satellite, and outer-satellite groups. The velocity scale is Local Standard of Rest, here and elsewhere.

dominated by relatively hot dust associated with the W 40 complex, where $\mathrm{NH}_{3}$ emission is present at a lower level. Weaker $\mathrm{NH}_{3}$ emission extending further to the south and north follows the color Herschel infrared image. Our mapped area with highest Galactic longitudes, region 5 in Fig. 1, showing only weak emission, is also seen in our $\mathrm{NH}_{3}(1,1)$ map. Overall, the $\mathrm{NH}_{3}$ distribution (Fig. 3) shows a ring-like morphology, with the center of this ring located in region 4 (see Fig. 1 and Sect.4.5). With a radius of $25^{\prime}-30^{\prime}$ (about $3.5 \mathrm{pc}$ ) it is roughly circular and exhibits comparatively strong dust and $\mathrm{NH}_{3}$ emission at its low and weak emission at its high Galactic longitude side.

Previous SPIRE/PACS observations from the Herschel Gould Belt survey towards the Aquila cloud complex (Könyves et al. 2015) identified 446 candidate prestellar cores and 58 protostellar cores in the Aquila region. Using this catalog, we find 362 prestellar cores and 49 protostellar cores in our observed area and show their distribution in Fig. B.1. The two data sets of pre- and protostellar cores on the one side and the $\mathrm{NH}_{3}$ data on the other match each other very well. All protostellar cores are associated with regions of notable $\left(>0.13 \mathrm{~K} \mathrm{~km} \mathrm{~s}^{-1}\right)$ $\mathrm{NH}_{3}$ emission, while prestellar cores are even found in the weak high-longitude region of the large ring-like structure.

Using the " $\mathrm{NH}_{3}(1,1)$ " fit procedure (see Sect.2.2), the $\mathrm{NH}_{3}(1,1)$ intensity-weighted mean velocity (moment 1 ) and velocity dispersion (moment 2) maps are presented in Fig. 4. 

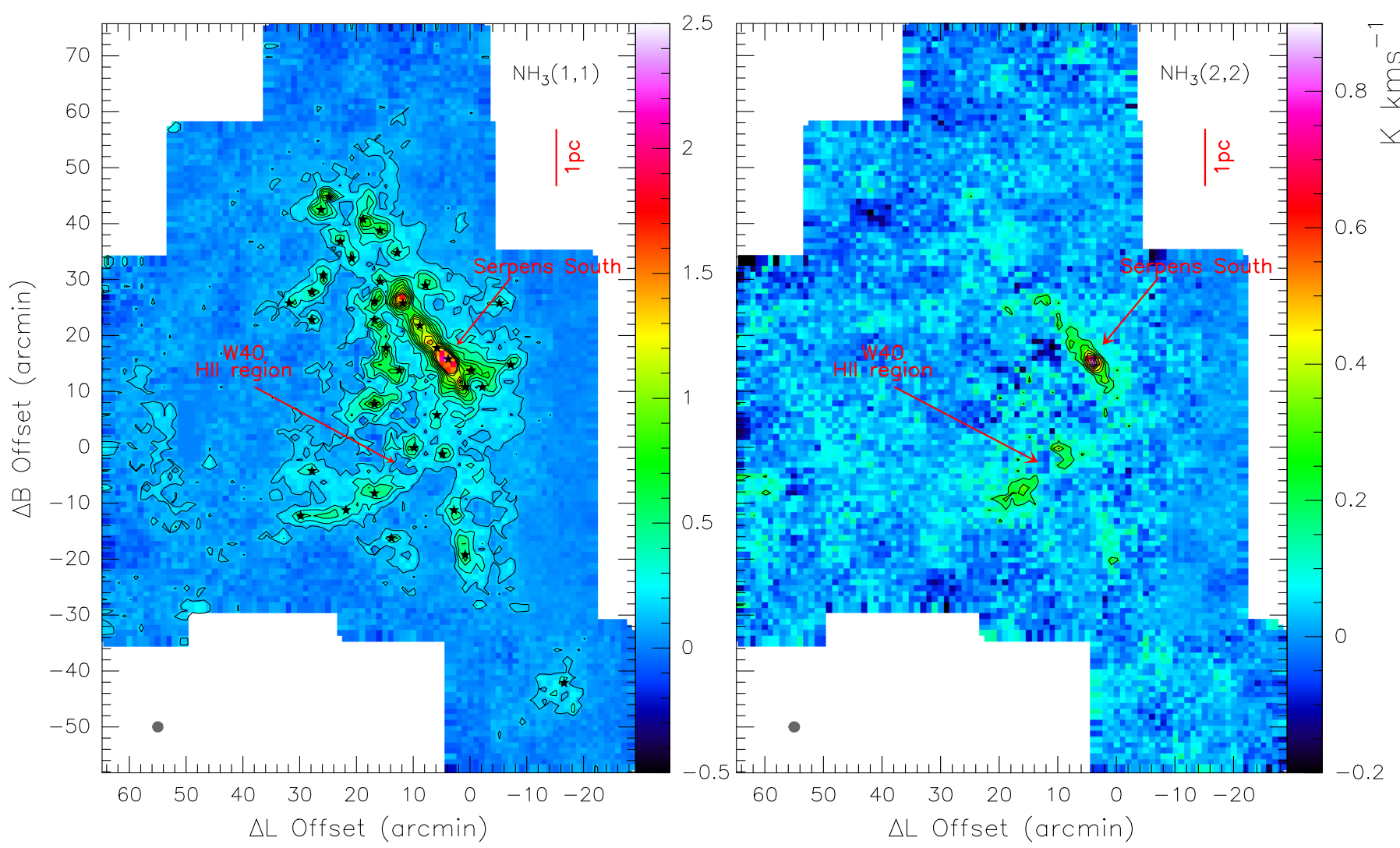

Fig. 3. Integrated intensity maps of $\mathrm{NH}_{3}(1,1)$ (left) and $(2,2)$ (right). The reference position is $l=28.59^{\circ}, b=3.55^{\circ}$. The integration range is $4<V_{\mathrm{LSR}}<10 \mathrm{~km} \mathrm{~s}^{-1}$. Contours start at $0.13 \mathrm{~K} \mathrm{~km} \mathrm{~s}^{-1}(3 \sigma)$ on a main beam brightness temperature scale and go up in steps of $0.13 \mathrm{~K} \mathrm{~km} \mathrm{~s}$. The unit of the color bars is $\mathrm{K} \mathrm{km} \mathrm{s}^{-1}$. The half-power beam width is illustrated as a black filled circle in the lower left corners of the images. The red line in the top right of each map illustrates the $1 \mathrm{pc}$ scale at a distance of $436 \mathrm{pc}$ (Ortiz-León et al. 2017, 2018). Left panel: black stars show the positions of the identified 38 ammonia clumps (see Sect. 3.2).

These maps visualize the kinematics of the Aquila Rift derived from $\mathrm{NH}_{3}(1,1)$. The two images are overlaid with $\mathrm{NH}_{3}(1,1)$ integrated intensity contours as in Fig. 3. Here we use a higher threshold of $5 \sigma$ for the lowest contours to provide reliable results. Previous $\mathrm{NH}_{3}$ observations with the GBT (beam size $30^{\prime \prime}$; Friesen et al. 2016) toward the Serpens South indicated narrow line widths, typically of order $0.5 \mathrm{~km} \mathrm{~s}^{-1}$, but with minima near $0.15 \mathrm{~km} \mathrm{~s}^{-1}$, and therefore in this case we used a velocity resolution of $0.1 \mathrm{~km} \mathrm{~s}^{-1}$ to fit the $\mathrm{NH}_{3}$ spectral lines. The intensity-weighted mean velocity map (see Fig. 4 left panel) reveals that the $\mathrm{mg}$ of $\mathrm{hf}$ components shows a velocity range from 4.5 to $8.4 \mathrm{~km} \mathrm{~s}^{-1}$. The dominant ridge of $\mathrm{NH}_{3}$ emission mostly indicates velocities in excess of $7 \mathrm{~km} \mathrm{~s}^{-1}$. Only at its southern edge and to the west of its northern edge velocities are smaller (see also Fig. 4 of Friesen et al. 2016). Velocities in regions 5 and 6 cannot be shown because the S/Ns are low. In these regions with weak $\mathrm{NH}_{3}(1,1)$ line emission we obtain averaged radial velocities of $8.2 \pm 0.8$ and $7.1 \pm 0.5 \mathrm{~km} \mathrm{~s}^{-1}$, respectively, which is consistent with those encountered in the regions with stronger emission.

From the FWHM line-widths (moment 2) map (see Fig. 4 right panel) we can see that the small region with widest lines is located in the northwest of Serpens South, while another small spot with relatively high dispersion is encountered in the south. Elsewhere, rather low and also uniform intrinsic (for individual hf components) dispersions $\left(\sigma \sim 0.5 \mathrm{~km} \mathrm{~s}^{-1}\right)$ are present. Irregular and larger dispersions are obtained in region 2 (see Fig. 1 for location and extent of this region). However, this may be merely a consequence of low S/Ns. We study the FWHM line widths of the $\mathrm{NH}_{3}(1,1)$ main lines with a peak line flux threshold of $5 \sigma$ as summarized in Fig. 5a. The line width refers to the individual hf components. The line width distribution of the $\mathrm{NH}_{3}(1,1)$ gas appears to have an outstanding peak around $0.5 \mathrm{~km} \mathrm{~s}^{-1}$, which is in agreement with the findings of Friesen et al. (2016).

\subsection{Dense clump identification}

Based on the distribution of the integrated intensity of the $\mathrm{NH}_{3}(1,1)$ line in Fig. 3, we use the Clumpfind2d algorithm (Williams et al. 1994) to identify dense core clumps. First, the root mean square of the integrated intensity map was derived based on a background noise estimate of $0.04 \mathrm{~K}$ in a $0.2 \mathrm{~km} \mathrm{~s}^{-1}$ wide channel from regions with weak emission. The level range from a threshold of 5 to $37 \sigma$ was then set with increments of $4 \sigma$ (where $\sigma$ is the rms noise level). Within the integrated intensity map, 38 potential dense clumps associated with the Aquila Rift were identified after eliminating a fake clump located at the boundary of the map. In order to further confirm the authenticity of the condensations identified by Clumpfind2d, we also tried different parameter settings and found that the location of the dense clumps remained the same and also that many false structure results appeared when using a lower threshold of $\leq 3 \sigma$. Adopting the output from the Clumpfind $2 \mathrm{~d}$ algorithm, it should be noted that the clumps identified around Aquila are located mostly along the dominant $\mathrm{NH}_{3}$ ridge with some additional sources further in the north and south (see Fig. 3). The black 

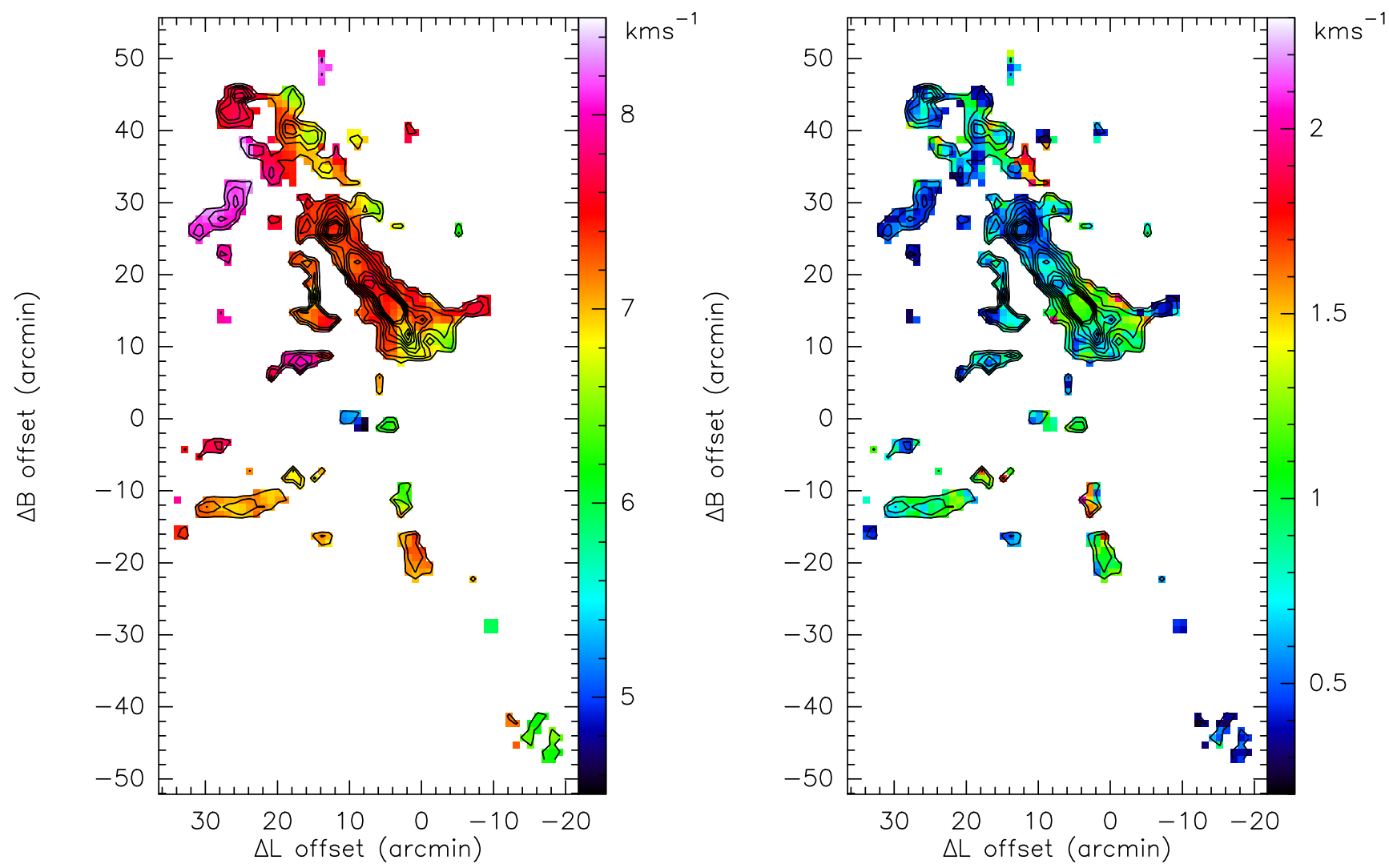

Fig. 4. Para- $\mathrm{NH}_{3}(1,1)$ intensity-weighted mean velocity (moment 1, left) and FWHM line width (moment 2, right) maps. The reference position is $l=28.59^{\circ}, b=3.55^{\circ}$. These intensity-weighted mean velocities and FWHM line widths referring to the individual hf components are derived from the GILDAS built-in " $\mathrm{NH}_{3}(1,1)$ " fitting method (Sect. 2.2). The considered velocity range is $4<V_{\mathrm{LSR}}<10 \mathrm{~km} \mathrm{~s}^{-1}$ for each panel. Contours of integrated intensity start at $0.23 \mathrm{~K} \mathrm{~km} \mathrm{~s}^{-1}(5 \sigma)$ on a main beam brightness temperature scale and go up in steps of $0.23 \mathrm{~K} \mathrm{~km} \mathrm{~s}^{-1}$.

stars in Fig. 3 (left panel) show the positions of the identified 38 clumps. Among these 38 clumps both $\mathrm{NH}_{3}(1,1)$ and $(2,2)$ emission lines are detected in 17 clumps. Observed parameters and calculated model parameters are given in Tables B.1-B.3. $\mathrm{NH}_{3}(1,1)$ and $(2,2)$ spectral lines towards the 17 clumps are shown in Fig. B.2. For the other 21 clumps $\mathrm{NH}_{3}(1,1)$ spectra are shown in Fig. B.3. Clumps are closely associated with pre- and protostellar cores. While all protostellar cores appear near the clumps, the prestellar cores exhibit a more widespread distribution (see also Fig. B.1 and Sect. 3.1).

\subsection{Kinetic temperature}

The relative population of the $K=1$ and 2 ladders of $\mathrm{NH}_{3}$ are highly sensitive to collisional processes because they are not directly connected radiatively. This allows us to use them as a thermometer of the gas kinetic temperature. We obtained the rotation temperature of $\mathrm{NH}_{3}(1,1)$ and $(2,2)$ using the method described in Ho \& Townes (1983), which is

$T_{\text {rot }}=\frac{-41.5}{\ln \left(\frac{-0.282}{\tau_{\mathrm{m}}(1,1)} \ln \left(1-\frac{T_{\mathrm{MB}}(2,2)}{T_{\mathrm{MB}}(1,1)}\left(1-\exp \left(-\tau_{\mathrm{m}}(1,1)\right)\right)\right)\right)} \mathrm{K}$,

where $\tau_{\mathrm{m}}$ is the peak optical depth of the $(1,1)$ main group of hf components derived using the GILDAS built-in " $\mathrm{NH}_{3}(1,1)$ " fitting method. The main beam brightness temperatures $T_{\mathrm{MB}}$ of the $(1,1)$ and $(2,2)$ inversion transitions were derived using the GILDAS built-in "GAUSS" fitting. A histogram of the peak optical depth of the $(1,1)$ main group of hyperfine components, $\tau_{\mathrm{m}}(1,1)$, for those positions with $\mathrm{NH}_{3}(1,1) S / N>5 \sigma$ is summarized in Fig. 5b. The optical depth distribution of the $\mathrm{NH}_{3}(1,1)$ gas peaks around 1.2. The $\mathrm{NH}_{3}(1,1)$ main beam brightness temperatures $\left(T_{\mathrm{MB}}<2 \mathrm{~K}\right)$ are higher than those of the $\mathrm{NH}_{3}(2,2)$ line $\left(T_{\mathrm{MB}}<0.5 \mathrm{~K}\right)$, meaning that the $\mathrm{NH}_{3}(2,2)$ lines can be considered to be optically thin.

In the most prominent regions, where both the $\mathrm{NH}_{3}(1,1)$ and $(2,2)$ lines were detected at levels of at least $5 \sigma$, the rotational temperature lies between 8.6 and $25.3 \mathrm{~K}$ with an average of $13.4 \pm 4.1 \mathrm{~K}$ (errors are standard deviations of the mean throughout the article). The median and mean values are 11.8 and $13.4 \mathrm{~K}$, respectively. The statistical distribution of $T_{\text {rot }}$ is summarized in Fig. $5 \mathrm{c}$ and shows a typical value of about $10 \mathrm{~K}$.

Following Tafalla et al. (2004) to connect rotational with kinetic temperatures, we used

$T_{\text {kin }}=\frac{T_{\text {rot }}(1,2)}{1-\frac{T_{\text {rot }}(1,2)}{42} \ln \left(1+1.1 \exp \left(\frac{-16}{T_{\text {rot }}(1,2)}\right)\right)} \mathrm{K}$,

where the energy gap between the $(1,1)$ and $(2,2)$ states is $\Delta E_{12}=42 \mathrm{~K}$. Tafalla et al. (2004) ran different Monte Carlo models involving the $\mathrm{NH}_{3}(J, K)=(1,1),(2,1)$, and $(2,2)$ inversion doublets and an $n(r)=n_{0} /\left(1+\left(r / r_{o}\right)^{2.5}\right)$ density distribution to compare their observationally determined approximately constant rotational temperatures with modeled kinetic temperatures in dense quiescent molecular clouds. Equation (2) is derived from fitting $T_{\text {kin }}$ in the range of 5 to $20 \mathrm{~K}$, and most of our sources can be found in this interval.

The kinetic temperatures of the dense gas in the Aquila Rift complex obtained from $\mathrm{NH}_{3}(2,2) /(1,1)$ ratios wherever the $(2,2)$ 

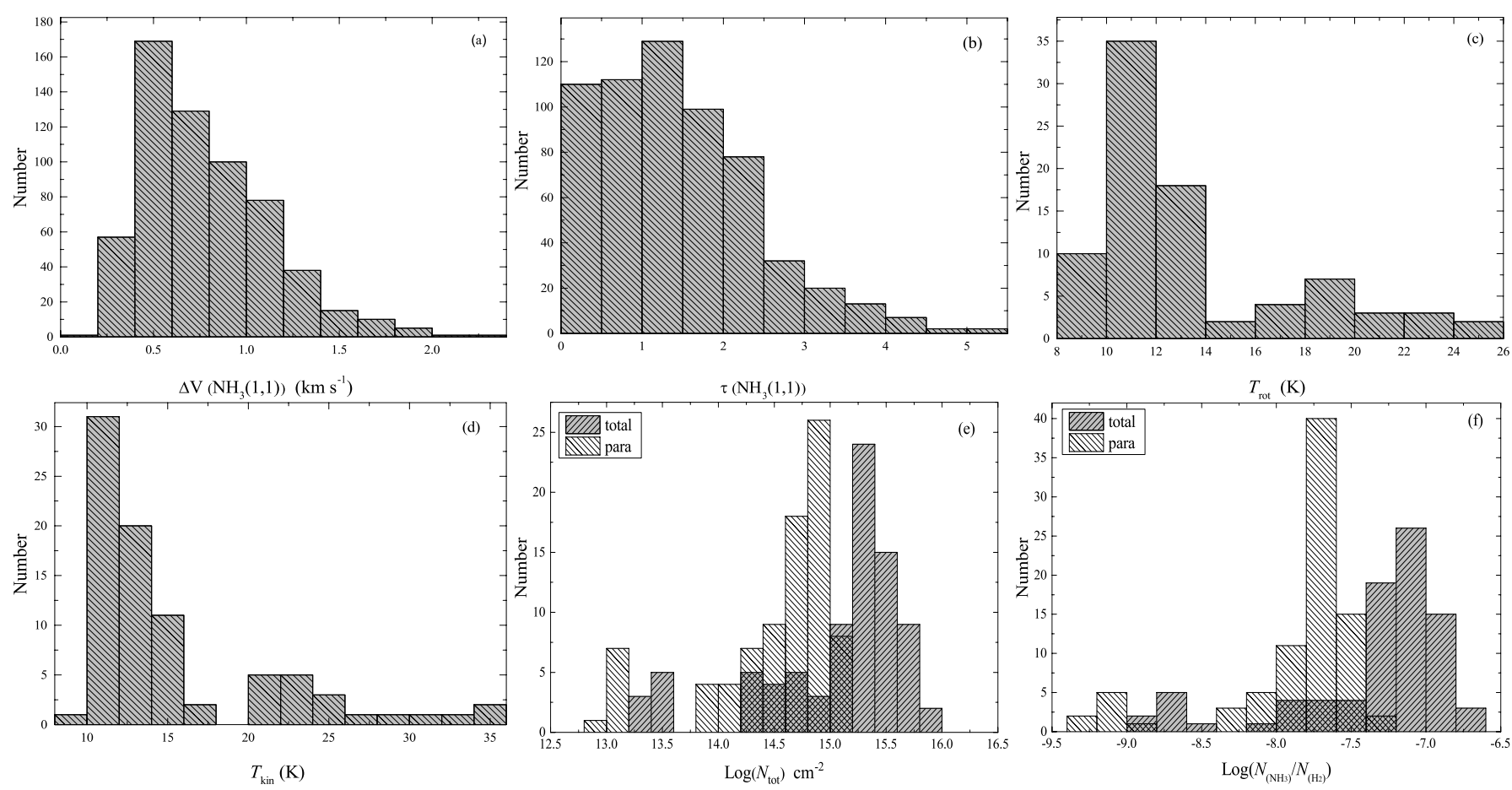

Fig. 5. Histograms of physical parameters derived from $\mathrm{NH}_{3}$. (a) Intrinsic $\mathrm{FWHM}$ line widths of individual $\mathrm{NH}_{3}(1,1)$ hyperfine structure components with a peak line flux threshold of $5 \sigma ;(b)$ peak optical depths of the main group of hf components $\tau_{\mathrm{m}}(1,1)$ for those positions with $\mathrm{NH}_{3}(1,1)$ $S / N s>5 \sigma$ (these line widths and peak optical depths are derived from the GILDAS built-in " $\mathrm{NH}_{3}(1,1)$ " fitting method); (c) rotational temperature $T_{\text {rot }}$, where both the $\mathrm{NH}_{3}(1,1)$ and $(2,2)$ lines were detected, at levels of at least $5 \sigma ;(d)$ kinetic temperature $T_{\text {kin }}$, for those positions with $\mathrm{NH}_{3}(2,2)$ line detections and $>5 \sigma$ features; (e) para and total (para+ortho) column densities of $\mathrm{NH}_{3}$, for those positions exhibiting $\mathrm{NH}_{3}(2,2)$ line detections and $>5 \sigma$ features, assuming an ortho- to para-ratio in thermal equilibrium at the presently obtained kinetic temperature; $(f)$ para- $\mathrm{NH}_{3}$ fractional abundance $\chi=\left[\right.$ para- $\left.\mathrm{NH}_{3}\right] /\left[\mathrm{H}_{2}\right]$, and total- $\mathrm{NH}_{3}$ (para+ortho) fractional abundance $\chi=\left[\right.$ total- $\left.\mathrm{NH}_{3}\right] /\left[\mathrm{H}_{2}\right]$, toward the positions with $\mathrm{NH}_{3}(2,2)$ line detections and $>5 \sigma$ features, again adopting ortho- to para $\mathrm{NH}_{3}$ ratios assuming thermal equilibrium.

line has been detected at a $>5 \sigma$ level range from 8.9 to $35.0 \mathrm{~K}$ with an average of $15.3 \pm 6.1 \mathrm{~K}$. The median and mean values are 12.7 and $15.3 \mathrm{~K}$, respectively. The distribution of the $\mathrm{NH}_{3}$ kinetic temperatures is presented in Fig. 5d and shows a typical value of about $12 \mathrm{~K}$.

The gas kinetic temperatures derived from the $\mathrm{NH}_{3}(2,2) /(1,1)$ map are shown in Fig. 6, left panel. We find that dense gas temperatures from para- $\mathrm{NH}_{3}$ in Serpens South are predominantly cold ranging from 8.9 to $16.8 \mathrm{~K}$ with an average of $12.3 \pm 1.7 \mathrm{~K}$, while W 40 at lower Galactic latitudes, which represents a young stellar cluster associated with an $\mathrm{H}$ in region, shows values from 17.7 to $35.0 \mathrm{~K}$ with an average of $25.1 \pm 4.9 \mathrm{~K}$ (see Table 1 for the relevant areas representing Serpens South and W 40). The kinetic temperatures in the dense gas around W 40 and in the lower right part of our map are high ( $25 \mathrm{~K}$; see Fig. 6 left panel), which is twice higher than that $(\sim 12 \mathrm{~K})$ in the LMSF region of Serpens South. For those 21 clumps with only upper limits to the $\mathrm{NH}_{3}(2,2)$ lines (see Sect. 3.2), the resulting upper kinetic temperature limits are $\lesssim 10 \mathrm{~K}$.

\section{4. $\mathrm{NH}_{3}$ column density}

The total para- $\mathrm{NH}_{3}$ column densities can be calculated from $\mathrm{NH}_{3}$ (1,1), following Wienen et al. (2012)

$$
\begin{aligned}
N_{\text {tot }} \approx & N(1,1)\left(\frac{1}{3} \exp \left(\frac{23.1}{T_{\text {rot }}(1,2)}\right)+1+\frac{5}{3} \exp \left(-\frac{41.2}{T_{\text {rot }}(1,2)}\right)\right. \\
& \left.+\frac{14}{3} \exp \left(-\frac{99.4}{T_{\text {rot }}(1,2)}\right)\right) \mathrm{cm}^{-2} .
\end{aligned}
$$

A178, page 6 of 19
In many cases the $\mathrm{NH}_{3}(1,1)$ inversion lines are optically thick, and the optical depth is determined by the GILDAS built-in " $\mathrm{NH}_{3}(1,1)$ " fitting method (see Sect. 2.2). The column density in the $(1,1)$ state is related to the optical depth $\tau_{\text {tot }}$, according to Mauersberger et al. (1986), by

$N(1,1)=\frac{1.65 \times 10^{14}}{v} \frac{J(J+1)}{K^{2}} \Delta v \tau_{\text {tot }} T_{\text {ex }} \mathrm{cm}^{-2}$,

where $N$ is in $\mathrm{cm}^{-2}$, the FWHM line width $\Delta v$ is in $\mathrm{km} \mathrm{s}^{-1}$, the line frequency $v$ is in $\mathrm{GHz}$, and the excitation temperature $T_{\mathrm{ex}}$ is in Kelvin. In the optically thick case, the excitation temperature $T_{\mathrm{ex}}$ is derived from the main beam brightness temperatures $T_{\mathrm{MB}}$ and the optical depth $\tau$ by

$T_{\mathrm{MB}}=\left(T_{\mathrm{ex}}-2.7 K\right)(1-\exp (-\tau)) \mathrm{K}$.

If $\tau \ll 1$, the main beam brightness temperature $T_{\mathrm{MB}}$ is assumed to be $T_{\text {ex }} \tau$.

We calculated the ortho-column densities using the orthoto para- $\mathrm{NH}_{3}$ abundance ratios as a function of $T_{\text {kin }}$ in Fig. 3 of Takano et al. (2002). These were added to the para-column density to get the total (para + ortho) column densities of $\mathrm{NH}_{3}$ (see Tables 2 and B.3).

The conversion between ortho- and para- $\mathrm{NH}_{3}$ is very slow and may take as long as $10^{6} \mathrm{yr}$ (Cheung et al. 1969). As a result, the excitation temperature between the ortho- and para-species, the so-called spin temperature, is often believed to reflect the formation temperature. Assuming thermalization in this sense, the ortho- to para- $\mathrm{NH}_{3}$ ratios are close to unity at $T_{\text {kin }} \gtrsim 30 \mathrm{~K}$, 
K. Tursun et al.: Ammonia observations towards the Aquila Rift cloud complex
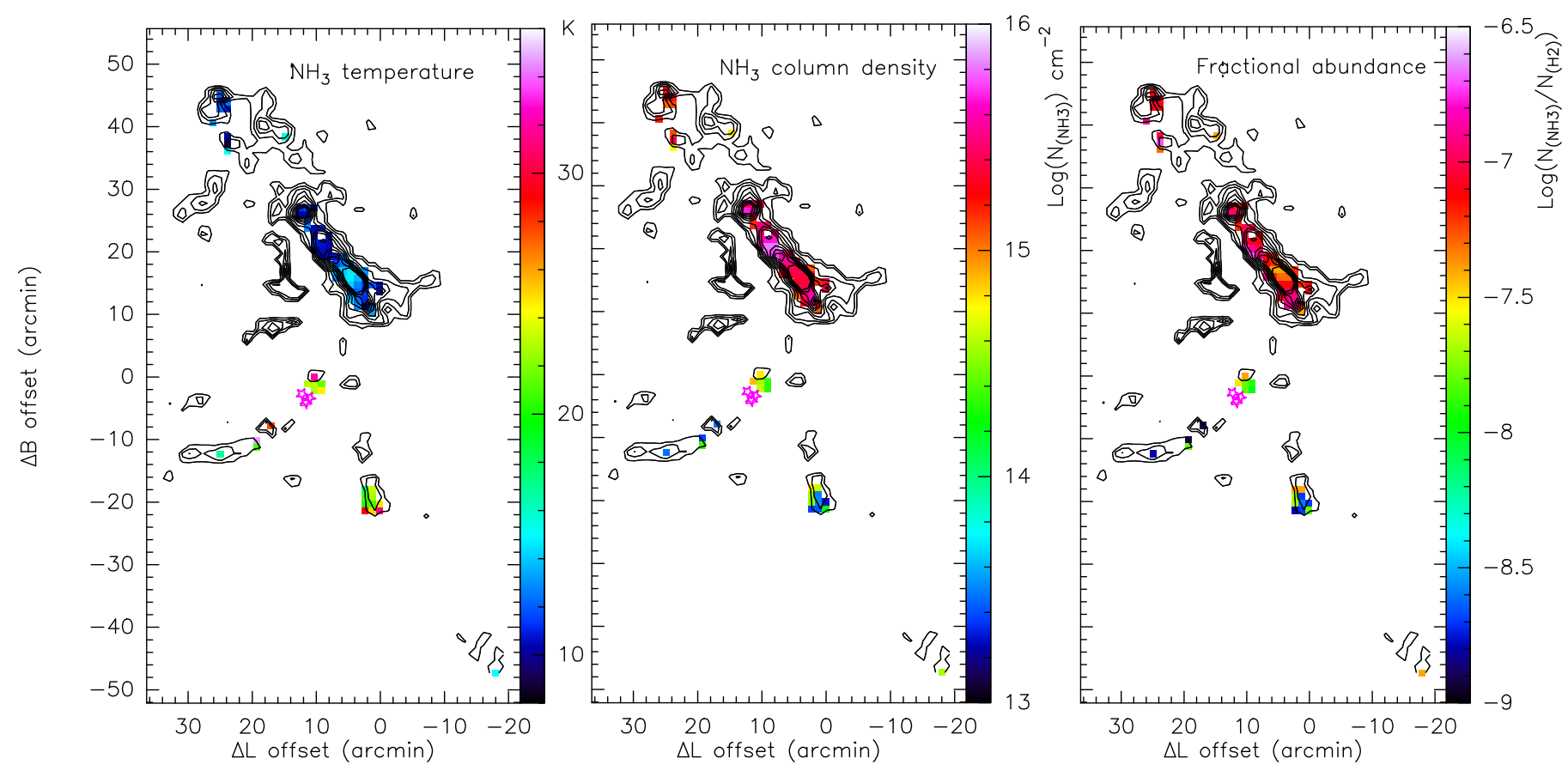

Fig. 6. Maps of $\mathrm{NH}_{3}$ kinetic temperature in units of Kelvin (left), the logarithm of the total- $\mathrm{NH}_{3}$ column density in units of $\mathrm{cm}^{-2}$ (middle), and the corresponding logarithm of the fractional abundance (right). The reference position is $l=28.59^{\circ}, b=3.55^{\circ}$. Contours of integrated $\mathrm{NH}_{3}(1,1)$ intensity are the same as in Fig. 4 and cover the velocity range $4<V_{\mathrm{LSR}}<10 \mathrm{~km} \mathrm{~s}^{-1}$. Contours start at $0.23 \mathrm{~K} \mathrm{~km} \mathrm{~s}^{-1}(5 \sigma)$ on a main beam brightness temperature scale and go up in steps of $0.23 \mathrm{~K} \mathrm{~km} \mathrm{~s}^{-1}$. Magenta stars show the locations of the OB association (OS1a, IRS1, IRS1a, IRS2, IRS2a, IRS2b, IRS3, and IRS3a) in W 40.

Table 2. Parameters obtained from Serpens South and W 40.

\begin{tabular}{|c|c|c|c|c|}
\hline \multirow[t]{2}{*}{ Parameter } & \multicolumn{2}{|c|}{ Serpens South } & \multicolumn{2}{|c|}{ W 40 ( $\mathrm{H}_{\text {II }}$ region) } \\
\hline & Range & Mean & Range & Mean \\
\hline Total- $N\left(\mathrm{NH}_{3}\right) / 10^{15} \mathrm{~cm}^{-2}$ & $0.03-6.4$ & $2.6 \pm 1.4$ & $0.02-0.8$ & $0.3 \pm 0.2$ \\
\hline$N\left(\mathrm{H}_{2}\right) / 10^{22} \mathrm{~cm}^{-2(a)}$ & $0.9-6.3$ & $3.2 \pm 1.4$ & $0.9-2.7$ & $1.7 \pm 0.5$ \\
\hline$\chi\left(\right.$ total $\left.-\mathrm{NH}_{3}\right) / 10^{-8}$ & $0.2-21.2$ & $8.6 \pm 3.8$ & $0.1-4.3$ & $1.6 \pm 1.4$ \\
\hline$T_{\text {gas }} / \mathrm{K}$ & $8.9-16.8$ & $12.3 \pm 1.7$ & $17.7-35.5$ & $25.1 \pm 4.9$ \\
\hline$T_{\text {dust }} / \mathrm{K}^{(b)}$ & $11.9-15.5$ & $13.4 \pm 0.9$ & $16.8-23.6$ & $19.1 \pm 2.2$ \\
\hline$v_{\mathrm{th}} / \mathrm{km} \mathrm{s}^{-1}$ & $0.09-0.12$ & $0.10 \pm 0.01$ & $0.13-0.18$ & $0.15 \pm 0.01$ \\
\hline$\sigma_{\mathrm{NT}} / \mathrm{km} \mathrm{s}^{-1}$ & $0.07-0.54$ & $0.32 \pm 0.12$ & $0.23-0.55$ & $0.41 \pm 0.08$ \\
\hline$c_{\mathrm{S}} / \mathrm{km} \mathrm{s}^{-1}$ & $0.18-0.25$ & $0.21 \pm 0.01$ & $0.25-0.36$ & $0.29 \pm 0.03$ \\
\hline$R_{\mathrm{P}}$ & $0.16-6.07$ & $0.70 \pm 0.90$ & $0.31-1.43$ & $0.57 \pm 0.27$ \\
\hline$M$ & $0.41-2.47$ & $1.51 \pm 0.50$ & $0.83-1.78$ & $1.39 \pm 0.23$ \\
\hline
\end{tabular}

Notes. ${ }^{(a)}$ Molecular hydrogen column densities and ${ }^{(b)}$ dust temperatures are taken from Bontemps et al. (2010) and Könyves et al. (2015). The errors shown are the standard deviations of the mean. For the areas adopted for Serpens South and W 40, see Table 1.

while they can reach values of four at $T_{\text {kin }} \sim 10 \mathrm{~K}$ (Takano et al. 2002). An even slightly higher range of uncertainty is introduced by detailed models also introducing uncertainties in the poorly known spin temperatures of $\mathrm{H}_{2}$ and $\mathrm{NH}_{4}^{+}$, which play an essential role in the formation of ammonia. Depending on the detailed circumstances, Faure et al. (2013) find ammonia ortho- to para abundance ratios of $\sim 1.5$ and even $\sim 0.7$, rather independent of kinetic temperature for values below $30 \mathrm{~K}$. We conclude that the maximum error in our adopted thermalized ortho-to-para $\mathrm{NH}_{3}$ abundance ratios could be a factor of $4.9 / 0.7=7.0$, representing a case where we determine $T_{\text {kin }} \sim 8.9 \mathrm{~K}$ (our lowest kinetic temperature), while the actual ortho- to para-abundance ratio is not 4.9 as expected in case of thermalization, but 0.7 (see Persson et al. 2012). However, even in this worst-case scenario our total
$\mathrm{NH}_{3}$ abundance would only be overestimated by half an order of magnitude, that is, by $5.9 / 1.7=3.5$. The higher the kinetic temperature, the smaller the uncertainty related to the ortho- $\mathrm{NH}_{3}$ column density correction.

The observed $\mathrm{NH}_{3}$ spectra were analyzed in the way described in Eqs. (1)-(5). In addition, detailed derivations of these equations are give in the Appendix of Pandian et al. (2012) and Levshakov et al. (2013). Furthermore, uncertainty estimations of the $\mathrm{NH}_{3}(1,1)$ and $(2,2)$ spectra are given in Appendix $\mathrm{C}$.

As can be seen in the central panel of Fig. 6, we provide a total column density map of $\mathrm{NH}_{3}$ for those positions exhibiting $\mathrm{NH}_{3}(2,2)$ line detections and $>5 \sigma \mathrm{NH}_{3}(1,1)$ features. The Aquila clumps show a broad distribution of total- $\mathrm{NH}_{3}$ column densities from $0.2 \times 10^{14}$ to $6.4 \times 10^{15} \mathrm{~cm}^{-2}$ with an 

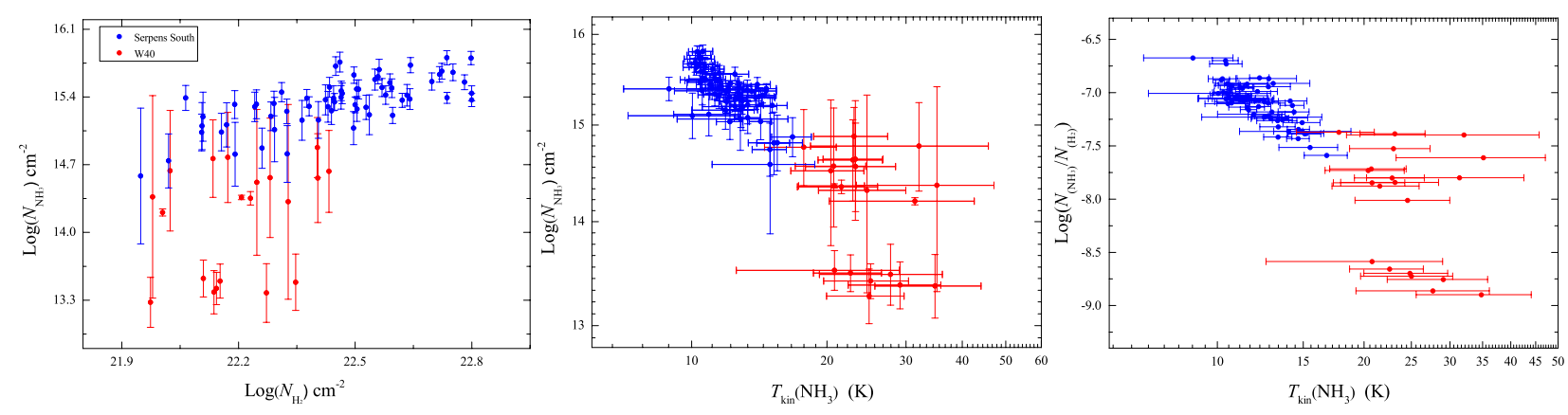

Fig. 7. Column densities derived from total- $N\left(\mathrm{NH}_{3}\right)$ for Serpens South (blue points) and $\mathrm{W} 40$ (red points) vs. $N\left(\mathrm{H}_{2}\right)$ column densities $($ left), total column densities of $N\left(\mathrm{NH}_{3}\right)$ vs. kinetic temperature (middle), and total fractional $\mathrm{NH}_{3}$ abundance, $N\left(\right.$ total- $\left.\mathrm{NH}_{3}\right) / N\left(\mathrm{H}_{2}\right)$, vs. kinetic temperature $T_{\text {kin }}\left(\mathrm{NH}_{3}\right)($ right $)$.

average of $2.1( \pm 1.6) \times 10^{15} \mathrm{~cm}^{-2}$. The total- $\mathrm{NH}_{3}$ column density range is $0.3 \times 10^{14}$ to $6.4 \times 10^{15} \mathrm{~cm}^{-2}$ with an average of $2.6( \pm 1.4) \times 10^{15} \mathrm{~cm}^{-2}$ in Serpens South, while in W 40 total $\mathrm{NH}_{3}$ column densities vary from 0.2 to $7.6 \times 10^{14} \mathrm{~cm}^{-2}$ with an average of $2.6( \pm 2.1) \times 10^{14} \mathrm{~cm}^{-2}$. The distribution of total column densities of $\mathrm{NH}_{3}$ is presented as a histogram in Fig. 5e and has an outstanding peak around $2.4 \times 10^{15} \mathrm{~cm}^{-2}$ seen in our data for Serpens South.

\section{Discussion}

\subsection{Variation of $\mathrm{NH}_{3}$ abundance}

Measurements of the $\mathrm{NH}_{3}$ abundance in different star-forming regions have shown a large spread, for example $10^{-5}$ in dense molecular "hot cores" around newly formed massive stars due to dust grain evaporation (Mauersberger et al. 1987), $10^{-8}$ in quiescent dark clouds (Benson \& Myers 1983), and $10^{-9}$ in the Orion Bar photon-dominated region (PDR; Batrla \& Wilson 2003; Larsson et al. 2003) because it is extremely affected by a high UV flux. A few times $10^{-10}$ in the Large Magellanic Cloud (LMC) and in M82 may characterize an environment with low metallicity and a high UV radiation field (Weiß et al. 2001; Ott et al. 2010).

Our total- $\mathrm{NH}_{3}$ column densities $N\left(\mathrm{NH}_{3}\right)$ are compared with the column densities of $\mathrm{H}_{2}$ derived from the Herschel infrared continuum data representing dust emission (André et al. 2010; Könyves et al. 2015), for which we smoothed the data to our beam size of $2^{\prime}$. The $\mathrm{NH}_{3}$ column densities are consistent with studies reported towards other Gould Belt star-forming regions (Friesen et al. 2017), where the logarithm of the para- $\mathrm{NH}_{3}$ column density $\left(\log N\left(\right.\right.$ para- $\left.\left.\mathrm{NH}_{3}\right)\right)$ varies from 13.0 to 15.5 .

The fractional total- $\mathrm{NH}_{3}$ abundance map $(\chi$ (total$\left.\mathrm{NH}_{3}\right)=\left(\right.$ total $\left.\left.-N\left(\mathrm{NH}_{3}\right)\right) / N\left(\mathrm{H}_{2}\right)\right)$ is shown in the right panel of Fig. 6. The relative total $\mathrm{NH}_{3}$ abundances $N\left(\right.$ total- $\left.\mathrm{NH}_{3}\right) / N\left(\mathrm{H}_{2}\right)$ range from $0.1 \times 10^{-8}$ to $2.1 \times 10^{-7}$ with an average of $6.9( \pm 4.5) \times 10^{-8}$, and the relative para- $\mathrm{NH}_{3}$ abundances $N($ para$\left.\mathrm{NH}_{3}\right) / N\left(\mathrm{H}_{2}\right)$ range from 0.1 to $4.3 \times 10^{-8}$ with an average of $1.8( \pm 0.9) \times 10^{-8}$ in our entire observed region (see Fig. 5f). Previous observations of $\mathrm{NH}_{3}$ in high-mass star-forming clumps suggest a median value of $N\left(\right.$ para- $\left.\mathrm{NH}_{3}\right) / N\left(\mathrm{H}_{2}\right)$ of $2.5 \times 10^{-8}$ (Urquhart et al. 2015). It addition, averaged $N\left(\right.$ para- $\left.\mathrm{NH}_{3}\right) / N\left(\mathrm{H}_{2}\right)$ values of $1.2 \times 10^{-7}, 4.6 \times 10^{-8}$, and $1.5 \times 10^{-8}$ were obtained by Dunham et al. (2011), Wienen et al. (2012), and Merello et al. (2019) in clumps of the Bolocam Galactic Plane Survey (BGPS), the APEX Telescope Large Area Survey of the GALaxy (ATLASGAL), and the Hi-GAL survey, respectively. Fractional abundances of $\sim 2-3 \times 10^{-8}$ were derived for protostellar and starless cores in Perseus, Taurus-Auriga, and infrared dark clouds (Tafalla et al. 2006; Foster et al. 2009; Chira et al. 2013).

The peak of our fractional para- $\mathrm{NH}_{3}$ abundance distribution lies slightly above the $10^{-8}$ range (see Fig. 5f). Friesen et al. (2016) found a factor of several variation in the para- $\mathrm{NH}_{3}$ abundance across Serpens South, with lowest detected abundances of $\chi\left(\right.$ para- $\left.\mathrm{NH}_{3}\right) \sim 5 \times 10^{-9}$, and highest abundances of $\sim 2 \times 10^{-8}$. We find para- $\mathrm{NH}_{3}$ abundances from 0.1 to $4.3 \times 10^{-8}$ with an average of $2.2( \pm 0.8) \times 10^{-8}$ in the same region. We also find that Serpens South shows total- $\mathrm{NH}_{3}$ fractional abundances ranging from $0.2 \times 10^{-8}$ to $2.1 \times 10^{-7}$ with an average of $8.6( \pm 3.8) \times 10^{-8}$. In W 40, the values are lower, namely between 0.1 and $4.3 \times 10^{-8}$ with an average of $1.6( \pm 1.4) \times 10^{-8}$, while we obtained fractional para- $\mathrm{NH}_{3}$ abundances ranging from 0.1 to $1.9 \times 10^{-8}$ with an average of $0.7( \pm 0.5) \times 10^{-8}$. These results imply that in $\mathrm{W} 40$, toward the positions with $\mathrm{NH}_{3}(2,2)$ line detections and $>5 \sigma \mathrm{NH}_{3}(1,1)$ features, total- $\mathrm{NH}_{3}$ abundances are a factor of approximately five lower than in Serpens South. Different stages of star formation apparently lead to different fractional $\mathrm{NH}_{3}$ abundances. The lower total- $\mathrm{NH}_{3}$ fractional abundance in W 40 compared to Serpens South is likely due to the fact that W 40 is strongly affected by FUV photons originating from the $\mathrm{H}$ il region. Ammonia is a particularly sensitive molecular species with respect to this kind of radiation (e.g., Weiß et al. 2001).

The total column densities of $\mathrm{NH}_{3}$, and its fractional abundances, $N\left(\right.$ total- $\left.\mathrm{NH}_{3}\right) / N\left(\mathrm{H}_{2}\right)$, as a function of $\mathrm{H}_{2}$ column density and kinetic temperature $T_{\text {kin }}\left(\mathrm{NH}_{3}\right)$ are shown in Fig. 7 . The total- $\mathrm{NH}_{3}$ column densities increase with $\mathrm{H}_{2}$ column densities in Serpens South, while there is no clear functional relation between total- $\mathrm{NH}_{3}$ column densities and $\mathrm{H}_{2}$ column densities in $\mathrm{W} 40$. However, in $\mathrm{W} 40$, low $N$ (total- $\mathrm{NH}_{3}$ ) values are only found in cases of low $\mathrm{H}_{2}$ column density (see Fig. 7, left panel). $N\left(\mathrm{H}_{2}\right)$ varies from 0.9 to $2.7 \times 10^{22} \mathrm{~cm}^{-2}$ with an average of $1.7( \pm 0.5) \times 10^{22} \mathrm{~cm}^{-2}$ in W 40. Serpens South is characterized by $N\left(\mathrm{H}_{2}\right)$ ranging from 0.9 to $6.3 \times 10^{22} \mathrm{~cm}^{-2}$ with an average of $3.2( \pm 1.4) \times 10^{22} \mathrm{~cm}^{-2}$ (see Table 2$)$. The total $\mathrm{NH}_{3}$ column densities and fractional abundances are inversely proportional to kinetic temperature in Serpens South (see Fig. 7 middle, right) and the entire surveyed region. However, no clear correlation with kinetic temperature is seen in W 40 alone.

\subsection{Comparison of gas and dust temperatures}

A comparison of gas kinetic temperatures derived from para$\mathrm{NH}_{3}(2,2) /(1,1)$ to HiGal dust temperatures (Bontemps et al. 2010 ) is shown in Fig. 8. The statistical distribution of $T_{\text {rot }}$ shows a typical value of about $10 \mathrm{~K}$. This is compatible with 

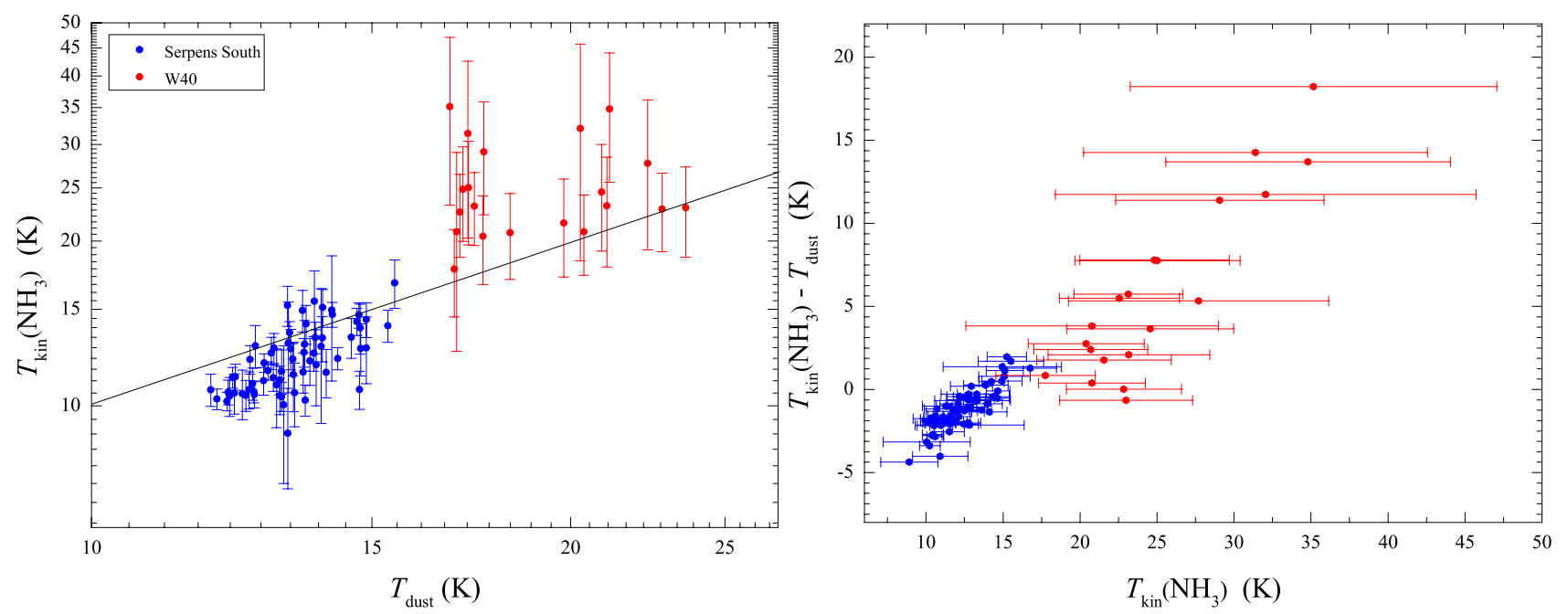

Fig. 8. Comparison of gas kinetic temperature derived from $\mathrm{NH}_{3}(2,2) /(1,1)$ ratios for Serpens South (blue points) and W 40 (red points) against dust temperature. The black line in the left panel indicates identical gas and dust temperature.

the characteristic kinetic temperature of local quiescent molecular gas, as indicated for example by Myers \& Benson (1983) using CO and by Myers \& Benson (1983) analyzing ammonia observations. The gas kinetic temperatures in the Serpens South region are similar to other active star-forming regions found by Friesen et al. (2017), such as Barnard 18 in Taurus $\left(T_{\text {kin }}=6-14 \mathrm{~K}\right)$, NGC 1333 in Perseus $\left(T_{\text {kin }}=8-21 \mathrm{~K}\right)$, and L1688 in Ophiuchus $\left(T_{\text {kin }}=9-25 \mathrm{~K}\right)$. The Orion A dense molecular cloud has been measured in $\mathrm{NH}_{3}(1,1)$ and $(2,2)$ with the GBT (Friesen et al. 2017). The typical gas kinetic temperature obtained from $\mathrm{NH}_{3}(2,2) /(1,1)$ is $20-30 \mathrm{~K}$. Measured gas kinetic temperatures are $>100 \mathrm{~K}$ in Orion KL, $>50 \mathrm{~K}$ in the Orion Bar, $\sim 50 \mathrm{~K}$ in Orion South, $20-30 \mathrm{~K}$ in the north of Orion molecular cloud 1 (OMC-1) and $>50 \mathrm{~K}$ in the northeastern part of the OMC-1 region (see Fig. 5 of Tang et al. 2018a based on $\mathrm{H}_{2} \mathrm{CO}$ data). The gas kinetic temperatures in the north of OMC-1 agree well with our results for the W 40 region. The dust temperatures of our sample are obtained from spectral energy distribution (SED) fitting to Herschel HiGal data at 70, 160, 250, 350, and $500 \mu \mathrm{m}$ by André et al. (2010) and Könyves et al. (2015). The dust temperatures derived on Herschel scales of $36^{\prime \prime}$ are smoothed to our beam size of $2^{\prime}$. In the region observed by us the dust temperatures range from 11.9 to $23.6 \mathrm{~K}$ with an average of $14.8 \pm 2.8 \mathrm{~K}$. Overall, the temperatures derived from para$\mathrm{NH}_{3}(2,2) /(1,1)$ tend to show higher temperatures than the HiGal dust temperatures.

Most of the clumps analyzed in this study lie in the optimal range of precise $T_{\text {kin }}$ determination when using $\mathrm{NH}_{3}(1,1)$ and $(2,2)$ lines (see Sects. 1 and 3.3). Figure 8 indicates that there is a large number of cold clumps with $T_{\text {kin }}<20 \mathrm{~K}$. The gas and dust are expected to be coupled at densities above about $10^{4.5}$ or $10^{5} \mathrm{~cm}^{-3}$ (Goldsmith 2001; Young et al. 2004). The temperatures derived from dust and gas are often in agreement in the active and dense clumps of Galactic disk clouds (Dunham et al. 2010; Giannetti et al. 2013; Battersby et al. 2014; Merello et al. 2019). This is also the case for Serpens South. Low gas temperatures are associated with Serpens South ranging from 8.9 to $16.8 \mathrm{~K}$ with an average of $12.3 \pm 1.7 \mathrm{~K}$, which is consistent with the mean value of $11 \pm 1 \mathrm{~K}$ found by Friesen et al. (2016). The gas and dust temperatures (mean and standard deviations $T_{\text {gas,avg }} \sim 12.3 \pm 1.7 \mathrm{~K}$ versus $T_{\text {dust,avg }} \sim 13.4 \pm 0.9 \mathrm{~K}$ ) scatter in Serpens South, but agree reasonably well as can be most directly seen in the right panel of Fig. 8 (blue points). However, in the HMSF region W 40, we find that the measured gas kinetic temperatures are higher than the dust temperatures (mean and standard deviations $T_{\text {gas,avg }} \sim 25.1 \pm 4.9 \mathrm{~K}$ versus $T_{\text {dust,avg }} \sim 19.1 \pm 2.2 \mathrm{~K}$ ), which indicates that the gas and dust are not well-coupled in W 40 and that the dust can cool more efficiently than the gas. This is consistent with the relatively weak $\mathrm{NH}_{3}$ lines associated with the core region of $\mathrm{W} 40$, indicating the presence of only small amounts of dense gas. This illustrates that the interplay between gas and dust cooling and heating is not uniform in the area covered by our observations. Such a difference between $T_{\text {gas }}$ and $T_{\text {dust }}$ is also seen in other regions and appears to be an often encountered property of massive-star formation regions. Battersby et al. (2014) and Koumpia et al. (2015) compare gas and dust temperatures in the massive-star-forming infrared dark cloud G32.02+0.05 and the high-mass-star-forming PDR S140, respectively, and find similar discrepancies between gas and dust temperatures. This likely indicates a lack of coupling between the gas and dust (Battersby et al. 2014) or could be due to the clouds being clumpy (Koumpia et al. 2015). These may be potential mechanisms relevant to $\mathrm{W} 40$, where the gas temperature is higher than the dust temperature.

\subsection{Thermal and nonthermal motions}

Previous observations of $\mathrm{NH}_{3}$ and $\mathrm{H}_{2} \mathrm{CO}$ in Galactic starforming regions (e.g., Wouterloot et al. 1988; Molinari et al. 1996; Jijina et al. 1999; Wu et al. 2006; Urquhart et al. 2011, 2015; Wienen et al. 2012; Lu et al. 2014; Tang et al. 2017, 2018a,b) suggest that the line width is correlated with kinetic temperature. Our findings imply that the correlation between line width and kinetic temperature is due to the dissipation of turbulent energy.

Here we examine whether there is a relationship between turbulence and temperature in our survey area. We computed thermal velocity $\left(v_{\mathrm{th}}\right)$, nonthermal velocity dispersion $\left(\sigma_{\mathrm{NT}}\right)$, and thermal sound speed $\left(c_{\mathrm{s}}\right) \cdot v_{\mathrm{th}}, \sigma_{\mathrm{NT}}$, and $c_{\mathrm{s}}$ are defined in Appendix A of Levshakov et al. (2014). For $v_{\text {th }}$ we have

$v_{\text {th }}=\sqrt{\frac{2 k_{\mathrm{B}} T_{\mathrm{kin}}}{m}} \mathrm{~km} \mathrm{~s}^{-1}$, 


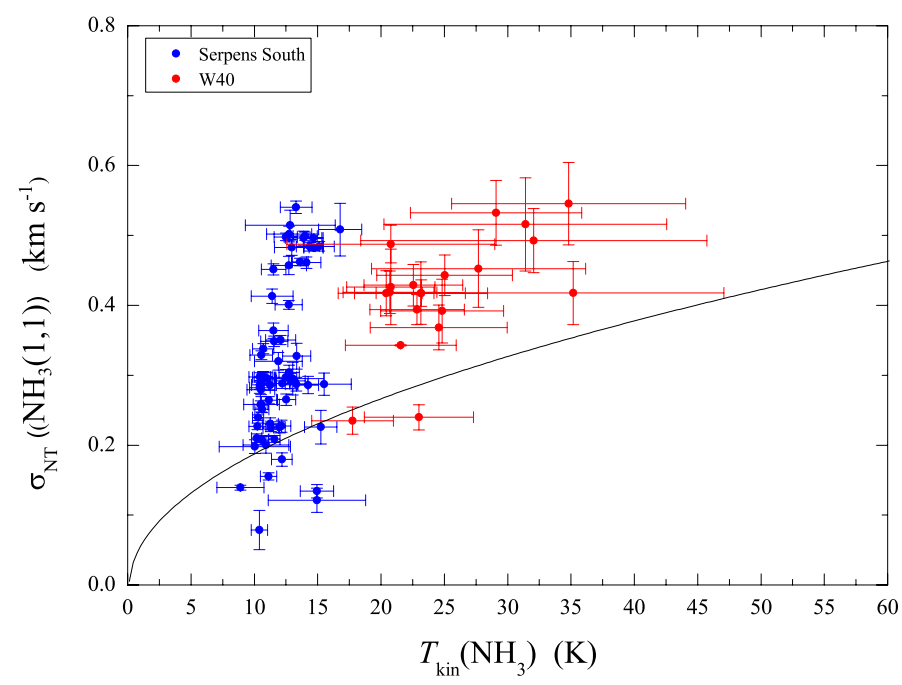

Fig. 9. Nonthermal velocity dispersion $\left(\sigma_{\mathrm{NT}}\right)$ vs. gas kinetic temperature derived from para- $\mathrm{NH}_{3}(1,1)$ for Serpens South (blue points) and W 40 (red points). The total dispersion of individual hyperfine structure (hfs) components are derived from the GILDAS built-in " $\mathrm{NH}_{3}(1,1)$ " fitting method for the $(1,1)$ line. The black lines represent the thermal sound speed.

where $k_{\mathrm{B}}$ is the Boltzmann constant, $m$ is the mass of a particle, and $T_{\text {kin }}$ is the kinetic temperature of the gas. For $\mathrm{NH}_{3}$, thermal velocity is

$v_{\text {th }}=0.03 \sqrt{T_{\text {kin }}} \mathrm{km} \mathrm{s}^{-1}$.

The thermal contribution to the observed line with $v_{\text {obs }}$ is related to the above-mentioned FWHM line width by (see Appendix C)

$v_{\mathrm{obs}}=\Delta v / 2 \sqrt{\ln 2} \mathrm{~km} \mathrm{~s}^{-1}$.

The $v_{\text {obs }}$ can be divided into a thermal and a turbulent part by

$v_{\mathrm{obs}}=\sqrt{v_{\mathrm{th}}^{2}+v_{\mathrm{turb}}^{2}} \mathrm{~km} \mathrm{~s}^{-1}$,

where $\Delta v$ is the FWHM line width of the $\mathrm{NH}_{3}(1,1)$ line obtained from the $\mathrm{NH}_{3}(1,1)$ fit in CLASS (see Sect. 2.2) and $\sigma_{\mathrm{NT}}$ is the nonthermal velocity dispersion along the line of sight:

$\sigma_{\mathrm{NT}}=v_{\mathrm{turb}} / \sqrt{2} \mathrm{~km} \mathrm{~s}^{-1}$.

This value can be compared with the thermal sound speed,

$c_{\mathrm{s}}=\sqrt{\frac{k_{\mathrm{B}} T_{\mathrm{kin}}}{\mu m_{\mathrm{H}}}} \mathrm{km} \mathrm{s}^{-1}$,

where $\mu=2.37$ is the mean molecular weight for molecular clouds (Dewangan et al. 2016) and $m_{\mathrm{H}}$ is the mass of the hydrogen atom. Comparisons of velocity dispersion and kinetic temperature are shown in Fig. 9. The thermal velocity of $\mathrm{NH}_{3}(1,1)$ for lines detected at a $>5 \sigma$ level ranges from 0.09 to $0.18 \mathrm{~km} \mathrm{~s}^{-1}$ with an average of $0.11 \pm 0.02 \mathrm{~km} \mathrm{~s}^{-1}$. The nonthermal velocity dispersion of $\mathrm{NH}_{3}(1,1)$ ranges from 0.07 to $0.55 \mathrm{~km} \mathrm{~s}^{-1}$ with an average of $0.34 \pm 0.12 \mathrm{~km} \mathrm{~s}^{-1}$. The derived nonthermal motions of $\mathrm{NH}_{3}$ are much higher than the thermal line widths of our survey area, implying that the line broadening of $\mathrm{NH}_{3}$ is dominated by nonthermal motions in these clumps. The BGPS sources which contain both starless and active star forming massive cores observed by Dunham et al. (2011) show average thermal velocity and nonthermal velocity dispersions of $0.12 \pm 0.02$ and $0.76 \pm 0.48 \mathrm{~km} \mathrm{~s}^{-1}$, respectively. Wienen et al. (2012) determined averages of $0.14 \pm 0.02$ and $0.90 \pm 0.40 \mathrm{~km} \mathrm{~s}^{-1}$ for thermal velocity and nonthermal velocity dispersions, respectively in the cold high-mass clumps of the ATLASGAL survey. The average thermal velocity of our $\mathrm{NH}_{3}(1,1)$ data agrees with previous results observed in massive star-forming clumps (Dunham et al. 2011; Wienen et al. 2012), but our nonthermal velocity dispersions are smaller than their values.

We also calculated the thermal to nonthermal pressure ratio $\left(R_{\mathrm{P}}=c_{\mathrm{S}}^{2} / \sigma_{\mathrm{NT}}^{2}\right.$; Lada et al. 2003) and Mach number (given as $\left.M=\sigma_{\mathrm{NT}} / c_{\mathrm{s}}\right)$. The sound speed ranges from 0.18 to $0.36 \mathrm{~km} \mathrm{~s}^{-1}$ with an average of $0.23 \pm 0.04 \mathrm{~km} \mathrm{~s}^{-1}$. The thermal to nonthermal pressure ratio in the gas traced by $\mathrm{NH}_{3}(1,1)$ ranges from 0.16 to 6.07 with an average of $0.67 \pm 0.79$. The BGPS sources observed by Dunham et al. (2011) show that the $R_{\mathrm{P}}$ values vary from 0.02 to 5.06 with an average of $0.20 \pm 0.33$. $\mathrm{NH}_{3}$ samples observed by Wienen et al. (2012) show values of $0.01-0.57$ with an average of $0.10 \pm 0.06$. Our average value for thermal to nonthermal pressure ratio is higher than previous results observed by Dunham et al. (2011) and Wienen et al. (2012). We find that the Mach number for $\mathrm{NH}_{3}(1,1)$ ranges from 0.41 to 2.47 with an average of $1.49 \pm 0.45$. The BGPS sources observed by Dunham et al. (2011) yield a mean Mach number of $3.2 \pm 1.8$. The average value of the Mach number we derive from $\mathrm{NH}_{3}$ may also be below the result $(3.4 \pm 1.1)$ of the various stages of high-mass star formation clumps with strong $\mathrm{NH}_{3}$ emission from the ATLASGAL survey (Wienen et al. 2012). Nevertheless, all this suggests that nonthermal pressure and supersonic nonthermal motions (e.g., turbulence, outflows, shocks, and/or magnetic fields) are dominant in the dense gas traced by $\mathrm{NH}_{3}$ in the Aquila region.

The derived values of $v_{\mathrm{th}}, \sigma_{\mathrm{NT}}, c_{\mathrm{s}}, R_{\mathrm{P}}$, and Mach number for Serpens South and W 40 are listed separately in Table 2. We calculated average nonthermal line widths of $\mathrm{NH}_{3}(1,1)$ for those positions with $\mathrm{NH}_{3}(1,1)>5 \sigma$ features in the subsamples consisting of Serpens South and W 40. For $\mathrm{NH}_{3}(1,1)$, the average nonthermal line widths $\sigma_{\mathrm{NT}}$ are $0.32 \pm 0.12 \mathrm{~km} \mathrm{~s}^{-1}$ and $0.41 \pm 0.08 \mathrm{~km} \mathrm{~s}^{-1}$, respectively, that is, quite similar. The average nonthermal line widths of $\mathrm{NH}_{3}(1,1)$ calculated here are consistent with those found in Serpens South by Friesen et al. (2016). These latter authors suggested that much of the dense gas in Serpens South has subsonic or trans-sonic nonthermal motions, while the mean $\sigma_{\mathrm{NT}}$ across the region is similar to the expected $\sim 0.2 \mathrm{~km} \mathrm{~s}^{-1}$ sound speed at $11 \mathrm{~K}$.

The similar nonthermal line widths between the $\mathrm{W} 40$ region and Serpens South are surprising. Either the entire region is already strongly affected by the consequences of massive star formation (e.g., through outflows and shocks) based on activity related to W 40 or the young low-mass stars in Serpens South are of sufficient number to induce turbulent motions to similar degree to that of the more massive stars in $\mathrm{W} 40$, which may have dissociated or expelled most of the dense molecular gas in their vicinity. Figure 9 shows that turbulent heating considerably contributes to gas temperature in these clumps.

\subsection{Radiative heating}

Previous SCUBA-2 450 and $850 \mu$ m observations of the W 40 complex in the Serpens-Aquila region (Rumble et al. 2016) provide evidence for radiative heating. The W 40 complex represents a HMSF region dominated by an $\mathrm{OB}$ association that is 


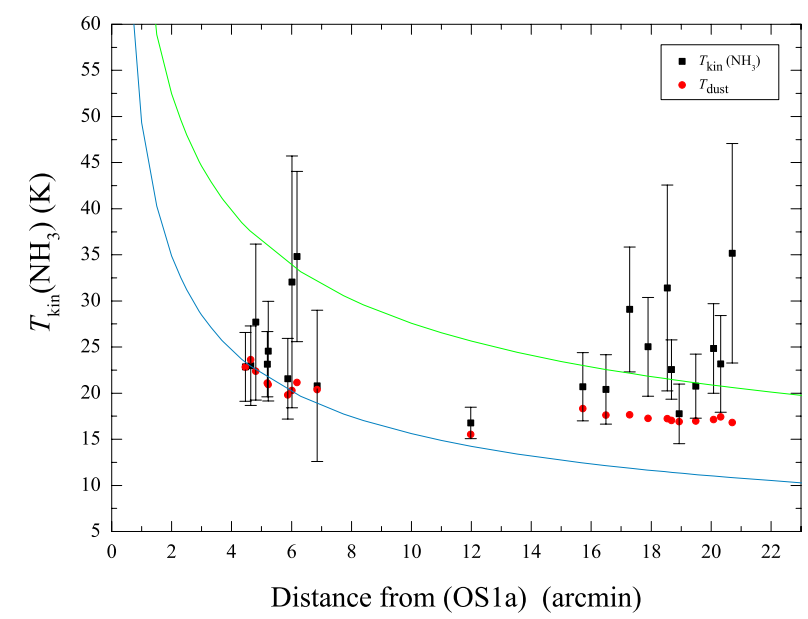

Fig. 10. Gas kinetic temperature derived from para- $\mathrm{NH}_{3}$ (black points) and dust temperature (red points) along the $\mathrm{W} 40$ region (projected distance from its main stellar source OS1 a, $l=28.79^{\circ}, b=3.49^{\circ}$ ). The blue and green lines are the expected relationships from a Stefan-Boltzmann law and modified Stefan-Boltzmann law (see Sect. 4.4), respectively, assuming OS1a is the dominant source with an approximate luminosity of $10^{5.25} L_{\odot}$ (Kuhn et al. 2010; Rumble et al. 2016).

powering an $\mathrm{H}$ II region. OS1a is the most luminous star in the W 40 complex (Rumble et al. 2016), but heating by the associated nearby $(D \leq 0.1 \mathrm{pc})$ sources IRS1, IRS1a, IRS2, IRS2a, IRS2b, IRS3, and IRS3a (their positions are indicated in Fig. 6) is also likely playing a role.

We investigate the relationship between gas kinetic temperature and projected distance $R$ from the central part of W 40 (OS 1a, $\left.l=28.79^{\circ}, b=3.49^{\circ}\right)$ in Fig. 10 . As is shown in the figure and as mentioned in Sect. 4.2, dust temperatures are lower than gas temperatures derived from $\mathrm{NH}_{3}$ in $\mathrm{W} 40$.

It is expected that the gas temperature and distance relation from the Stefan-Boltzmann blackbody radiation law is $T_{\text {kin }}=100 \times\left(\frac{7.4 \times 10^{-5}}{R}\right)^{\frac{1}{2}}\left(\frac{L}{L_{\odot}}\right)^{\frac{1}{4}} \mathrm{~K}$, adopting a molecular cloud distance of $436 \mathrm{pc}$ (Ortiz-León et al. 2017, 2018) and assuming that OS1a is the dominant source with an approximate luminosity of $10^{5.25} L_{\odot}$ (Kuhn et al. 2010; Wiseman \& Ho 1998). For the emissivity of the dust grains smaller than the wavelength at the characteristic blackbody temperature, the radiation law is adjusted to $T_{\text {kin }}=100 \times\left(\frac{1.2 \times 10^{-4}}{R}\right)^{\frac{2}{5}}\left(\frac{L}{L_{\odot}}\right)^{\frac{1}{5}} \mathrm{~K}$ (Wiseman \& Ho 1998).

The two radiation models for gas heating (Stefan-Boltzmann blackbody radiation and its modification related to dust emissivity) are both not well supported by our para- $\mathrm{NH}_{3}$ data (Fig. 10) which exclude positions related to Serpens South. Contrary to our expectation, temperatures near $\sim 18^{\prime}(\sim 2 \mathrm{pc})$ offsets appear to be slightly higher than those at $\sim 5^{\prime}$ offsets. This holds for temperatures derived by both the gas and dust and is not in agreement with the declining dust temperatures found by Rumble et al. 2016 (their Fig. 15) as a function of distance from the main stellar source of $\mathrm{W} 40$. For $\mathrm{NH}_{3}$ this may imply that the dense molecular gas in the vicinity of $\mathrm{W} 40$ has been destroyed by UV radiation (Sect. 4.1) and that the projected angular distances in Fig. 10 may be significantly below the real distances. In any case, dust grain mantle evaporation as seen in hot cores (e.g., in Orion-KL) and leading to very high ammonia column densities is not seen. W 40 might have gone through such a phase of evolution, but UV photons may have destroyed its short-lived chemical consequences (for models, see e.g., Charnley et al. 1992) in the meantime. As discussed in Sect.4.1, Serpens South shows a fractional total$\mathrm{NH}_{3}$ abundance with an average of $8.6( \pm 3.8) \times 10^{-8}$. In W 40, values are lower $1.6( \pm 1.4) \times 10^{-8}$, which is a factor of $\sim 5$ below the result from Serpens South.

The nature of the molecular ridge, which is not particularly pronounced in the dust continuum maps of Fig. 1 but dominates the maps of $\mathrm{NH}_{3}$ emission in Figs. 3, 4, and 6, is still poorly defined. It could represent a PDR similar to the Orion bar northwest of the Trapezium stars (Tang et al. 2018a), or swept up material near the edge of an expanding $\mathrm{H}$ II region. Since radial velocities (see Fig. 4) do not hint at a significant difference between W 40 and Serpens South, such material would have been swept up mostly along the plane of the sky. Additional molecular surveys addressing the detailed chemistry of this region could shed more light on this puzzle.

\subsection{Kinematics of the dense gas}

To study the velocity pattern of our observed area in more detail, we fitted the velocities of the entire measured region, Serpens South, and W 40. There may be weak velocity gradients that run along the entire observed region, Serpens South, and W 40 (see left panel of Fig. 4). Following the steps of Goodman et al. (1993) and $\mathrm{Wu}$ et al. (2018), we fitted the velocity adopting a linear form: $V_{\mathrm{LSR}}=v_{0}+a \times \Delta l+b \times \Delta b$, where $v_{0}$ is the systemic velocity of the cloud, and $\Delta l$ and $\Delta b$ are offsets in Galactic longitude and Galactic latitude. The velocity gradient can be derived as $\nabla v=\left(a^{2}+b^{2}\right)^{0.5} / D$, where distance $D=436 \mathrm{pc}$ (Sect. 1). This distance was used for all spectra with peak line fluxes larger than $5 \sigma$. This linear velocities fitting is based on solid body approximations for Serpens South and W 40, Serpens South alone, and W 40 alone (see Table 1 for the adopted extent of those regions). The derived gradients are then $0.16 \pm 0.01$, $0.27 \pm 0.01$, and $0.38 \pm 0.01 \mathrm{~km} \mathrm{~s}^{-1} \mathrm{pc}^{-1}$ for the observed region shown in Fig. D.1, Serpens South, and W 40, respectively.

To check the assumption of solid body rotation we study the fitted velocity and velocity residuals $\left(V_{\mathrm{obs}}-V_{\text {fit }}\right)$ in Appendix D for the region encompassing both Serpens South and W 40. The black solid polyline indicates a potential rotation axis $(0.019 \Delta l+$ $0.007 \Delta b-0.328=0$, in arcmin) in the fitted velocity map of the entire observed region (Fig. D.1 left panel), and three black parallel lines perpendicular to this rotation axis show the direction of the velocity gradient. Velocities tend to be lower in the lower right of the left panel of Fig. D.1. For Serpens South alone (see Table 1 for the extent of the region), the rotation axis is $0.03 \Delta l-0.01 \Delta b-0.12=0$, and for $\mathrm{W} 40$ it is $0.04 \Delta l-0.03 \Delta b-0.88=0$ arcmin. From Fig. D.1, we can see that most of the velocity residuals are distributed in the range $V_{\text {obs }}-V_{\text {fit }}=-1$ to $1 \mathrm{~km} \mathrm{~s}^{-1}$ which is even more clearly shown in Fig. D.2. To be specific, $90 \%$ of the velocity residuals of the observed region shown in Fig. D.1, Serpens South, and W 40 are in the range $V_{\mathrm{obs}}-V_{\text {fit }}<0.65,0.56$, and $0.79 \mathrm{~km} \mathrm{~s}^{-1}$, respectively. Larger velocity residuals are mainly located, in terms of Galactic coordinates, at the western and northeastern part of Serpens South and the southern part of W 40.

A maximum velocity gradient of $0.38 \pm 0.01 \mathrm{~km} \mathrm{~s}^{-1} \mathrm{pc}^{-1}$ for W 40 can be considered as an upper limit for an estimate of the rotational energy. Taking Serpens South and W 40 as a rigidly rotating cylinder, the ratio of rotational to gravitational energy is given (Bonnell et al. 1992; Wu et al. 2018) by

$$
\beta=\frac{E_{\mathrm{rot}}}{E_{\text {grav }}}=\frac{1}{2} \frac{M L^{2}}{12}\left(1+\frac{3}{2 x^{2}}\right) \omega^{2} / \frac{3}{2} \frac{G M^{2}}{L} f(x) \approx \frac{\omega^{2}}{4 \pi G \rho} \frac{L^{2}}{9 R^{2}},
$$


where $G$ is the gravitational constant and $\rho, L, R$, and $\omega$ are the density, height, radius, and the angular velocity of the cylinder. This can be simplified to

$\beta=\frac{3.0 \times 10^{-3} \omega_{-14}^{2}}{n_{4}} \frac{L^{2}}{9 R^{2}}$,

where, $\omega_{-14}$ is the angular velocity in units of $10^{-14} \mathrm{~s}^{-1}$ and the gas density $n_{4}$ is in units of $10^{4} \mathrm{~cm}^{-3}$ (Levshakov et al. 2013). For the entire observed region, Serpens South, and W 40, we adopt angular velocities, which are equal to the velocity gradients calculated before, $0.51,0.88$, and $1.23 \times 10^{-14} \mathrm{~s}^{-1}$ and an average density of the molecular Aquila complex of $10^{4} \mathrm{~cm}^{-3}$ (Levshakov et al. 2013). From Eq. (13), the ratio of rotational to gravitational energy for the entire observed region, Serpens South, and W 40 we obtained are $\beta \lesssim 0.003,0.01$, and 0.02 , respectively. It is clear that in the entire observed region, Serpens South, and W 40, rotation is presently playing a negligible role. The rotational energy is a very small fraction of the gravitational energy.

Using parallax measurements taken with the Very Large Baseline Array (VLBA), Ortiz-León et al. (2017, 2018) obtain distances slightly in excess of $400 \mathrm{pc}$ for both Serpens South and the W 40 region, which together show an arc-like morphology (see Figs. 1, 3, B.1). However, including the weaker region 5 (Fig. 1) and the high and low Galactic latitude regions of region 4 (again Fig. 1), we encounter a ring-like morphology, possibly forming a shell of gas. One important question is whether or not the weakly emitting high-Galactic-longitude part of this ring is located at a similar distance to that obtained by Ortiz-León et al. (2017, 2018). While this remains an open question, we note that this high-longitude part of the putative shell shows about the same radial velocities as Serpens South and W 40 (Sect. 3.1). With respect to any Galactic kinematical model, clouds with similar radial velocity and not more than one degree apart should be located at similar distances.

While we cannot prove that similar kinematic distances correspond in our case to a physical connection, it is nevertheless worthwhile to analyze the observed structure with this assumption, noting that a similar morphology is also seen in ${ }^{13} \mathrm{CO}$ (which likely traces lower density gas than $\mathrm{NH}_{3}$ ) in Fig. 10 of $\mathrm{Su}$ et al. (2020) and in the $\mathrm{H}_{2}$ column density map of Könyves et al. (2010). The ring-like large-scale morphology of both the dust (Fig. 1) and the ${ }^{13} \mathrm{CO}$ - and $\mathrm{NH}_{3}$-emitting gas (Fig. 15 of $\mathrm{Su}$ et al. 2019 and our Figs. 3 and B.1) is reminiscent of an expanding interstellar bubble with large amounts of dense gas on one side and lesser amounts of such gas on the other.

Since velocities in regions 1 and 5 (see Fig. 1) are similar (Sect. 3.1), they might represent those parts of the putative shell that are expanding parallel to the plane of the sky. However this would mean that velocities inside the rim of this structure should be different, more redshifted on the back and more blueshifted on the front side. For all three rotation axes crossing the low-Galactic-longitude side of the shell-like structure, lower velocities and blueshifted areas are located at lower Galactic longitudes, while higher velocities and redshifted areas are located at higher longitudes. This is compatible with a radial motion of the entire shell-like structure and would indicate slow expansion, if the gas at higher longitudes forms a part of the back side of the shell, or slow contraction, if the gas is part of the front side of the shell. The velocity difference is about $0.7 \mathrm{~km} \mathrm{~s}^{-1}$.

For the kinematic age of the putative expanding shell, with

$t=\sqrt{\left(r_{x} \times r_{y}\right) / v_{\text {exp }}}$, $r_{x}$ and $r_{y} \sim 3.5 \mathrm{pc}$, and $v_{\exp }=0.7 \mathrm{~km} \mathrm{~s}^{-1}$ we obtain a timescale of several million years. However, we note that the $0.7 \mathrm{~km} \mathrm{~s}^{-1}$ are for this approach only a lower limit: (1) we do not see gas along the center of the putative shell, where the discrepancy in velocity relative to its rim should be largest; (2) the expansion of the gas may have significantly slowed down due to entrainment of ambient gas originally not participating in this expansion.

In this context it may be worth noting that the center of the putative shell is not known to host a supernova remnant (W. Reich, priv. comm.). More sensitive data, in particular from the high-Galactic-longitude parts of the ring-like structure (see Figs. 1 and 3) would be helpful to further investigate related scenarios.

\section{Summary}

We mapped the western part of the Aquila Rift cloud complex in the $\mathrm{NH}_{3}(1,1)$ and $(2,2)$ transitions, which includes the densest regions of Serpens South and W 40. The main results of this work can be summarized as follows.

1. The $\mathrm{NH}_{3}$ morphology, revealing the distribution of the dense gas $\left(n\left(\mathrm{H}_{2}\right) \gtrsim 10^{-3} \mathrm{~cm}^{-3}\right)$, is correlated with that of the Herschel infrared dust emission and shows an overall similar structure with two exceptions: there is, in Galactic coordinates, the well-known NE-SW ridge of dense gas, which forms the core of the Serpens South region and is much more dominant in $\mathrm{NH}_{3}$ than in the dust emission. W 40 dominates the dust emission but is much less pronounced in $\mathrm{NH}_{3}$. The ridge with strong $\mathrm{NH}_{3}(1,1)$ emission contains several cores along a total length of about $15^{\prime}$. Weaker emission extends further to lower and higher Galactic latitudes and toward regions at larger longitudes. Overall, the $\mathrm{NH}_{3}$ distribution forms, like the dust, a circle with a diameter of $50^{\prime}-60^{\prime}$ $(\sim 7 \mathrm{pc})$, with comparatively strong emission at low and weak emission at high longitudes.

2. The kinetic temperature of the dense gas in the Aquila rift cloud complex measured by $\mathrm{NH}_{3}(2,2) /(1,1)$ line ratios ranges from 8.9 to $35.0 \mathrm{~K}$ with an average of $15.3 \pm 6.1 \mathrm{~K}$. The HMSF region of $\mathrm{W} 40$ has a gas kinetic temperature of $\sim 25 \mathrm{~K}$, which is twice that $(\sim 12 \mathrm{~K})$ found in the LMSF region of Serpens South.

3. Fractional abundances of total $\mathrm{NH}_{3}$ vary from $0.1 \times 10^{-8}$ to $2.1 \times 10^{-7}$ with an average of $6.9( \pm 4.5) \times 10^{-8}$. Serpens South has total $\mathrm{NH}_{3}$ fractional abundances also ranging from $0.2 \times 10^{-8}$ to $2.1 \times 10^{-7}$ with an average of $8.6( \pm 3.8) \times 10^{-8}$. Lower values of $0.1-4.3 \times 10^{-8}$ with an average of $1.6( \pm 1.4) \times 10^{-8}$ characterize $\mathrm{W} 40$, which is a factor of approximately five below the result from Serpens South.

4. A comparison of kinetic temperatures derived from $\mathrm{NH}_{3}$ and dust emission indicates that gas and dust temperatures are in agreement in Serpens South, but gas temperatures are higher than those of the dust in W 40. This suggests that gas and dust are coupled in Serpens South, but not in W 40.

5. Dense gas traced by $\mathrm{NH}_{3}$ is significantly influenced by supersonic nonthermal motions. Similar levels of nonthermal turbulence are encountered in W 40 and Serpens South. This may either be caused by the fact that the entire region is already strongly affected by the consequences of massive star formation based on activity related to W 40 or that a similar amount of turbulence is triggered by the large number of forming low-mass stars in Serpens South. W 40 appears to have dissociated or expelled most dense molecular gas in its vicinity. 
K. Tursun et al.: Ammonia observations towards the Aquila Rift cloud complex

6. The nonthermal velocity dispersion of $\mathrm{NH}_{3}(1,1)$ is positively correlated with the gas temperature, which indicates that the dense gas may be heated by dissipation of turbulent energy.

7. Velocity gradients of $0.16 \pm 0.01,0.27 \pm 0.01$, and $0.38 \pm 0.01 \mathrm{~km} \mathrm{~s}^{-1} \mathrm{pc}^{-1}$ appear to be present in the observed region shown in Fig. D.1, Serpens South alone, and W 40 alone, respectively. The rotational and gravitational energy of the Aquila region are compared using those velocity gradients. For the entire observed region, Serpens South, and $\mathrm{W} 40$, ratios are about $\beta \lesssim 0.003,0.01$, and 0.02 , respectively. This demonstrates that the rotational energy is a negligible fraction of the gravitational energy.

8. The morphology of the entire studied region can be described by a ring or shell with a diameter of about $7 \mathrm{pc}$ and strong emission at lower Galactic longitudes and weak emission at higher Galactic longitudes. However, this only holds in a physical sense, if all parts are located at approximately the same distance. This is presently only known for the lower-Galactic-longitude part with its strong emission. While we find velocity gradients in the radial direction at the low-longitude side of the putative shell, it is therefore not yet clear whether this indicates a systematic expansion (or contraction) of the entire structure.

Acknowledgements. We would like to thank the anonymous referee for the useful suggestions that improved this study. The authors are thankful for helpful comments by Wolfgang Reich. We thank the staff of the Nanshan 26-m radio telescope for their assistance during the observations. This work is based on measurements made with the Nanshan 26-m radio telescope, which is operated by the Key Laboratory of Radio Astronomy, Chinese Academy of Sciences. This work was funded by the National Natural Science foundation of China under grant 11433008, 11903070, 11603063, 11703074, 11703073, and 11973076, the Heaven Lake Hundred-Talent Program of Xinjiang Uygur Autonomous Region of China, and the CAS "Light of West China" Program under Grant 2018-XBQNXZB-024, 2016-QNXZ-B-23, and 2016-QNXZ-B-22. C.H. acknowledges suppor by a Chinese Academy of Sciences President's International Fellowship Initiative for visiting scientists (2021VMA0009). This research has used NASA's Astrophysical Data System (ADS).

\section{References}

André, P., Men'shchikov, A., Bontemps, S., et al. 2010, A\&A, 518, L102 Barranco, J. A., \& Goodman, A. A. 1998, ApJ, 504, 207

Batrla, W., \& Wilson, T. L. 2003, A\&A, 408, 231

Battersby, C., Bally, J., Dunham, M., et al. 2014, ApJ, 786, 116

Benson, P. J., \& Myers, P. C. 1983, ApJ, 270, 589

Bonnell, I., Arcoragi, J.-P., Martel, H., et al. 1992, ApJ, 400, 579

Bontemps, S., André, P., Könyves, V., et al. 2010, A\&A, 518, L85

Charnley, S. B., Tielens, A. G. G. M., \& Millar, T. J. 1992, ApJ, 399, L71

Cheung, A. C., Rank, D. M., Townes, C. H., et al. 1969, ApJ, 157, L13

Chira, R.-A., Beuther, H., Linz, H., et al. 2013, A\&A, 552, A40

Danby, G., Flower, D. R., Valiron, P., et al. 1988, MNRAS, 235, 229

Dewangan, L. K., Ojha, D. K., Luna, A., et al. 2016, ApJ, 819, 66

Dunham, M. K., Rosolowsky, E., Evans, N. J., II, et al. 2010, ApJ, 717, 1157

Dunham, M. K., Rosolowsky, E., Evans, N. J., II, et al. 2011, ApJ, 741, 110

Dobashi, K., Uehara, H., Kandori, R., et al. 2005, PASJ, 57, S1

Evans, N. J., II 1999, ARA\&A, 37, 311

Faure, A., Hily-Blant, P., Le Gal, R., et al. 2013, ApJ, 770, L2

Foster, J. B., Rosolowsky, E. W., Kauffmann, J., et al. 2009, ApJ, 696, 298

Friesen, R. K., Bourke, T. L., Di Francesco, J., Gutermuth, R., Myers, P. C. 2016 ApJ, 833, 204

Friesen, R. K., Pineda, J. E., co-PIs, et al. 2017, ApJ, 843, 63
Giannetti, A., Brand, J., Sánchez-Monge, Á., et al. 2013, A\&A, 556, A16 Goodman, A. A., Benson, P. J., Fuller, G. A., et al. 1993, ApJ, 406, 528 Goldsmith, P. F. 2001, ApJ, 557, 736

Gutermuth, R. A., Bourke, T. L., Allen, L. E., et al. 2008, ApJ, 673, L151

Ho, P. T. P., \& Townes, C. H. 1983, ARA\&A, 21, 239

Hunter, J. D. 2007, Comput. Sci. Eng., 9, 90

Jijina, J., Myers, P. C., \& Adams, F. C. 1999, ApJS, 125, 161

Komesh, T., Esimbek, J., Baan, W., et al. 2019, ApJ, 874, 172

Könyves, V., André, P., Men'shchikov, A., et al. 2010, A\&A, 518, L106

Könyves, V., André, P., Men'shchikov, A., et al. 2015, A\&A, 584, A91

Koumpia, E., Harvey, P. M., Ossenkopf, V., et al. 2015, A\&A, 580, A68

Kuhn, M. A., Getman, K. V., Feigelson, E. D., et al. 2010, ApJ, 725, 2485

Lada, C. J., \& Lada, E. A. 2003, ARA\&A, 41, 57

Lada, C. J., Bergin, E. A., Alves, J. F., \& Huard, T. L. 2003, ApJ, 586, 286

Larsson, B., Liseau, R., Bergman, P., et al. 2003, A\&A, 402, L69

Levshakov, S. A., Henkel, C., Reimers, D., et al. 2013, A\&A, 553, A58

Levshakov, S. A., Henkel, C., Reimers, D., \& Wang, M. 2014, A\&A, 567, A78

Lu, X., Zhang, Q., Liu, H. B., Wang, J., \& Gu, Q. 2014, ApJ, 790, 84

Mallick, K. K., Kumar, M. S. N., Ojha, D. K., et al. 2013, ApJ, 779, 113

Mauersberger, R., Henkel, C., Wilson, T. L., \& Walmsley, C. M. 1986, A\&A, 162,199

Mauersberger, R., Henkel, C., \& Wilson, T. L. 1987, A\&A, 173, 352

Merello, M., Molinari, S., Rygl, K. L. J., et al. 2019, MNRAS, 483, 5355

Molinari, S., Brand, J., Cesaroni, R., \& Palla, F. 1996, A\&A, 308, 573

Myers, P. C., \& Benson, P. J. 1983, ApJ, 266, 309

Myers, P. C., Dame, T. M., Thaddeus, P., et al. 1986, ApJ, 301, 398

Nakamura, F., Dobashi, K., Shimoikura, T., Tanaka, T., \& Onishi, T. 2017, ApJ, 837,154

Ortiz-León, G. N., Dzib, S. A., Kounkel, M. A., et al. 2017, ApJ, 834, 143

Ortiz-León, G. N., Loinard, L., Dzib, S. A., et al. 2018, ApJ, 869, L33

Ott, J., Henkel, C., Staveley-Smith, L., et al. 2010, ApJ, 710, 105

Pandian, J. D., Wyrowski, F., \& Menten, K. M. 2012, ApJ, 753, 50

Persson, C. M., De Luca, M., Mookerjea, B., et al. 2012, A\&A, 543, A145

Rodríguez, L. F., Rodney, S. A., \& Reipurth, B. 2010, AJ, 140, 968

Rohlfs, K., \& Wilson, T. L. 2004, Tools of Radio Astronomy, eds. K. Rohlfs, \& T. L. Wilson (Berlin: Springer), 2004

Rumble, D., Hatchell, J., Pattle, K., et al. 2016, MNRAS, 460, 4150

Schreyer, K., Henning, T., Koempe, C., \& Harjunpaeae, P. 1996, A\&A, 306, 267

Shirley, Y. L. 2015, PASP, 127, 299

Smith, J., Bentley, A., Castelaz, M., et al. 1985, ApJ, 291, 571

Sokolov, V., Wang, K., Pineda, J. E., et al. 2017, A\&A, 606, A133

Su, Y., Yang, J., Zhang, S., et al. 2019, ApJS, 240, 9

Su, Y., Yang, J., Yan, Q.-Z., et al. 2020, ApJ, 893, 91

Suzuki, H., Yamamoto, S., Ohishi, M., et al. 1992, ApJ, 392, 551

Tafalla, M., Myers, P. C., Caselli, P., \& Walmsley, C. M. 2004, A\&A, 416, 191

Tafalla, M., Santiago-García, J., Myers, P. C., et al. 2006, A\&A, 455, 577

Takano, S., Nakai, N., \& Kawaguchi, K. 2002, PASJ, 54, 195

Tan, J. C., Beltrán, M. T., Caselli, P., et al. 2014, Protostars and Planets VI (Tucson, AZ: University of Arizona Press), 149

Tang, X.D., Henkel, C., Menten, K. M., et al. 2017, A\&A, 598, A30

Tang, X. D., Henkel, C., Menten, K. M., et al. 2018a, A\&A, 609, A16

Tang, X. D., Henkel, C., Wyrowski, F., et al. 2018b, A\&A, 611, A6

Ungerechts, H., Walmsley, C. M., \& Winnewisser, G. 1986, A\&A, 157, 207

Urquhart, J. S., Morgan, L. K., Figura, C. C., et al. 2011, MNRAS, 418, 1689

Urquhart, J. S., Figura, C. C., Moore, T. J. T., et al. 2015, MNRAS, 452, 4029

Vallee, J. P. 1987, A\&A, 178, 237

Walmsley, C. M., \& Ungerechts, H. 1983, A\&A, 122, 164

Weiß, A., Neininger, N., Henkel, C., Stutzki, J., \& Klein, U. 2001, ApJ, 554, L143

Wienen, M., Wyrowski, F., Schuller, F., et al. 2012, A\&A, 544, A146

Williams, J. P., de Geus, E. J., \& Blitz, L. 1994, ApJ, 428, 693

Wiseman, J. J., \& Ho, P. T. P. 1998, ApJ, 502, 676

Wouterloot, J. G. A., Walmsley, C. M., \& Henkel, C. 1988, A\&A, 203, 367

Wu, Y., Zhang, Q., Yu, W., et al. 2006, A\&A, 450, 607

Wu, G., Qiu, K., Esimbek, J., et al. 2018, A\&A, 616, A111

Young, K. E., Lee, J.-E., Evans, N. J., II, et al. 2004, ApJ, 614, 252

Zeilik, M., II, \& Lada, C. J. 1978, ApJ, 222, 896

Zinnecker, H., \& Yorke, H. W. 2007, ARA\&A, 45, 481 


\section{Appendix A: Calibration stability}

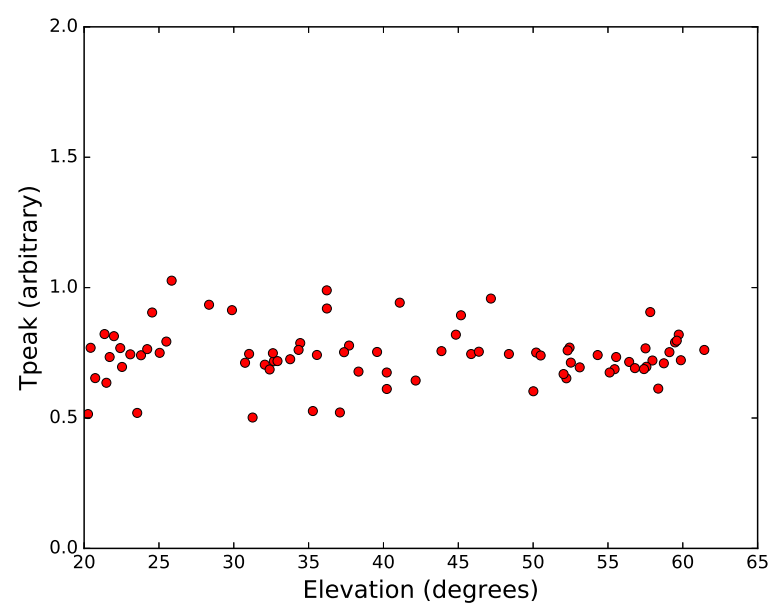

Fig. A.1. Uncorrected $\mathrm{NH}_{3}(1,1)$ main line intensities against elevation of repeated observations toward the reference position. The position corresponds to RA: 00:36:47.51, Dec: +63:29:02.1 (J2000). The standard deviations of the mean of the flux is about $10 \%$.

The system temperature was calibrated against a signal injected by a noise diode. Hot (ambient temperature) and cold (liquid nitrogen) loads determine the temperature of the noise diode. We observed the reference position (RA: 00:36:47.51, Dec: $+63: 29: 02.1$ with $(0,0)$ offset, J2000) to check the calibration stability every $2-3 \mathrm{~h}$. All reference position observations were made in the OTF mode of a small area of $6^{\prime} \times 6^{\prime}$. To present the peak distribution against elevation (Fig. A.1), we regridded the data and then fitted the $\mathrm{NH}_{3}(1,1)$ main lines (the central group of $\mathrm{NH}_{3}(1,1)$ hyperfine components). From Fig. A.1, we clearly see that there is no significant systematic variation. The standard deviations of the mean of the peak intensities is about $10 \%$, thus the observational system of the Nanshan observatory is stable.

To further check our calibration stability, the $\mathrm{NH}_{3}(1,1)$ data of G035.39-0.33 collected using the GBT (Sokolov et al. 2017) were used as a comparision with our $\mathrm{NH}_{3}$ data. For the process, the GBT data were smoothed to our beam size using the "XY_MAP" routine in GILDAS. In Fig. A.2, the GBT spectrum (black) and our spectrum (red) of the reference position (RA: 18:57:07.94, Dec: 02:10:51.40, J2000) are displayed. The two spectra can clearly be seen to match each other well and the checking results are reliable.

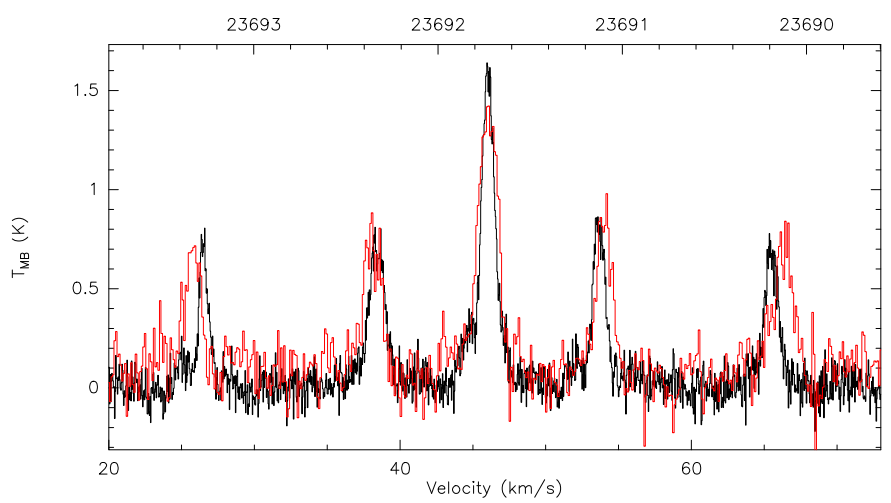

Fig. A.2. $\mathrm{NH}_{3}(1,1)$ spectra from the GBT (black) and our data (red) toward the reference position RA: 18:57:07.94, Dec: 02:10:51.40 (J2000).

Table A.1. Area name, central position, average noise level and covered size of the mapped regions (see also Fig. 1).

\begin{tabular}{cccc}
\hline \hline Area name & $\begin{array}{c}\text { Central position } \\
(l, b)\end{array}$ & $\begin{array}{c}\text { Area } \\
\text { degree } \times \text { degree }\end{array}$ & $\begin{array}{c}\text { Noise level } \\
\mathrm{K}\end{array}$ \\
\hline 1 & $\left(28.59^{\circ}, 3.55^{\circ}\right)$ & $0.7 \times 1.1$ & 0.05 \\
2 & $\left(28.75^{\circ}, 4.30^{\circ}\right)$ & $0.4 \times 0.4$ & 0.03 \\
3 & $\left(28.39^{\circ}, 2.81^{\circ}\right)$ & $0.5 \times 0.4$ & 0.04 \\
4 & $\left(29.19^{\circ}, 3.79^{\circ}\right)$ & $0.5 \times 1.4$ & 0.04 \\
5 & $\left(29.55^{\circ}, 3.54^{\circ}\right)$ & $0.2 \times 1.1$ & 0.05 \\
6 & $\left(28.87^{\circ}, 4.65^{\circ}\right)$ & $0.6 \times 0.3$ & 0.05 \\
\hline
\end{tabular}

\section{Appendix B: Prestellar and protostellar cores and $\mathrm{NH}_{3}$ spectra toward the Aquila Rift cloud complex and derived physical parameters}

In this appendix we present prestellar and protostellar cores taken from Könyves et al. (2015) (see Fig. B.1), and show the $\mathrm{NH}_{3}$ spectra for the 38 Clumps identified with Clumpfind2d in Sect. 3.2. The observed spectra of the $\mathrm{NH}_{3}(1,1)$ and $(2,2)$ transitions detected toward their peak positions are shown in Figs. B.2 and B.3. Measured physical parameters are listed in Tables B.1-B.3. 

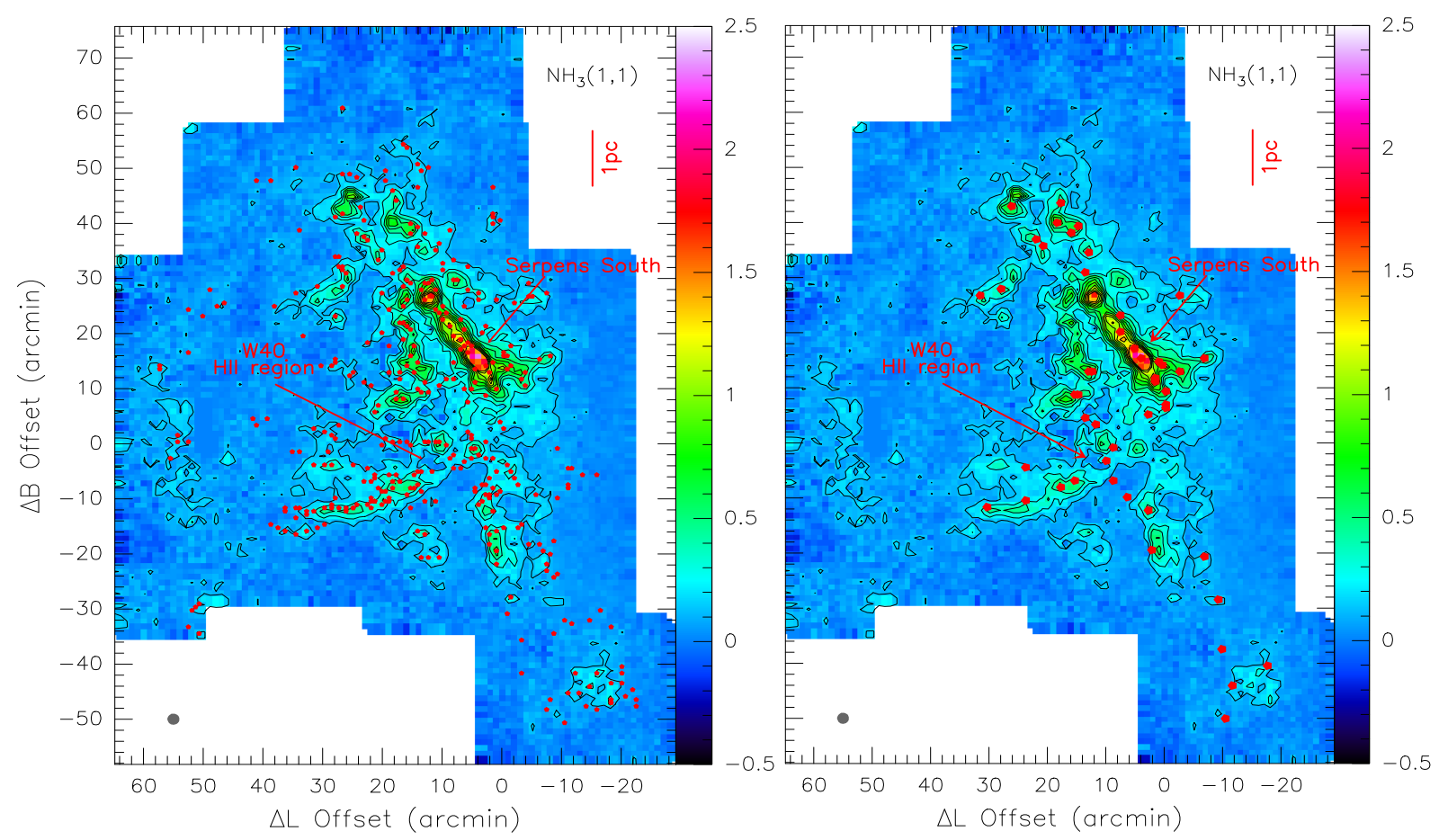

Fig. B.1. Integrated intensity maps of $\mathrm{NH}_{3}(1,1)$ (left and right). The reference position is $l=28.59^{\circ}, b=3.55^{\circ}$. The integration range is $4<V_{\mathrm{LSR}}<10 \mathrm{~km} \mathrm{~s}^{-1}$. Contours start at $0.13 \mathrm{~K} \mathrm{~km} \mathrm{~s}^{-1}(3 \sigma)$ on a main beam brightness temperature scale and go up in steps of $0.13 \mathrm{~K} \mathrm{~km} \mathrm{~s}{ }^{-1}$. The unit of the color bars is $\mathrm{K} \mathrm{km} \mathrm{s}^{-1}$. The half-power beam width is illustrated as a black filled circle in the lower left corners of the images. Left and right panels: the red points show the positions of the 362 candidate prestellar cores and 49 protostellar cores taken from Könyves et al. (2015), respectively. The red line in the top right of each map illustrates the $1 \mathrm{pc}$ scale at a distance of $436 \mathrm{pc}$ (Ortiz-León et al. 2017, 2018).
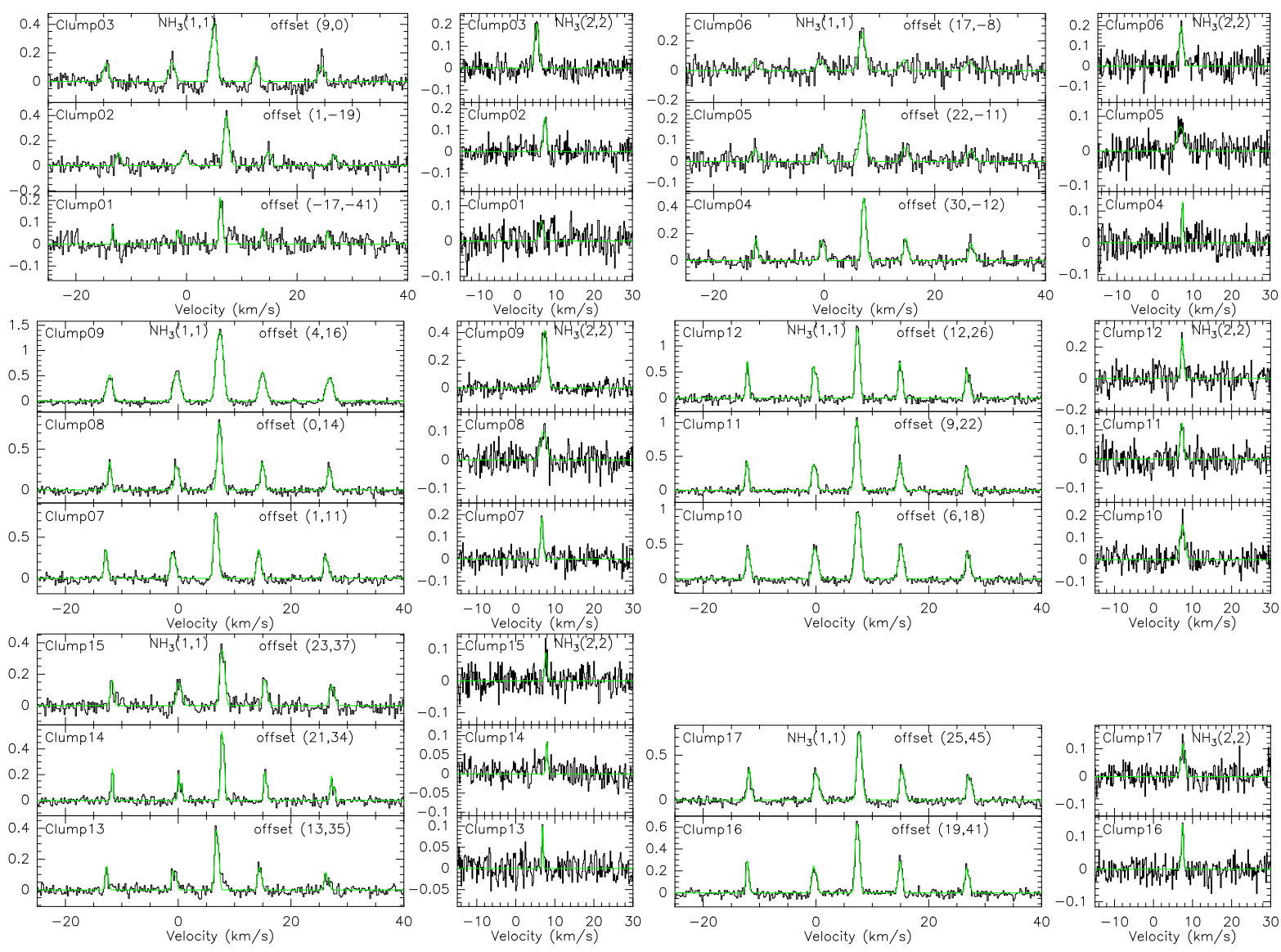

Fig. B.2. $\mathrm{NH}_{3}(1,1)$ and $(2,2)$ spectra towards clumps 01 to 17 . Green colour indicates the $\mathrm{NH}_{3}(1,1)$ fitting and Gaussian fitting of the $\mathrm{NH}_{3}(2,2)$ lines (see Sect. 2.2). The central position of this area is at $(l, b)=\left(28.59^{\circ}, 3.55^{\circ}\right)$. Offsets in Galactic coordinates (unit: arcmin) are shown in the top right corner of each $\mathrm{NH}_{3}(1,1)$ panel. 

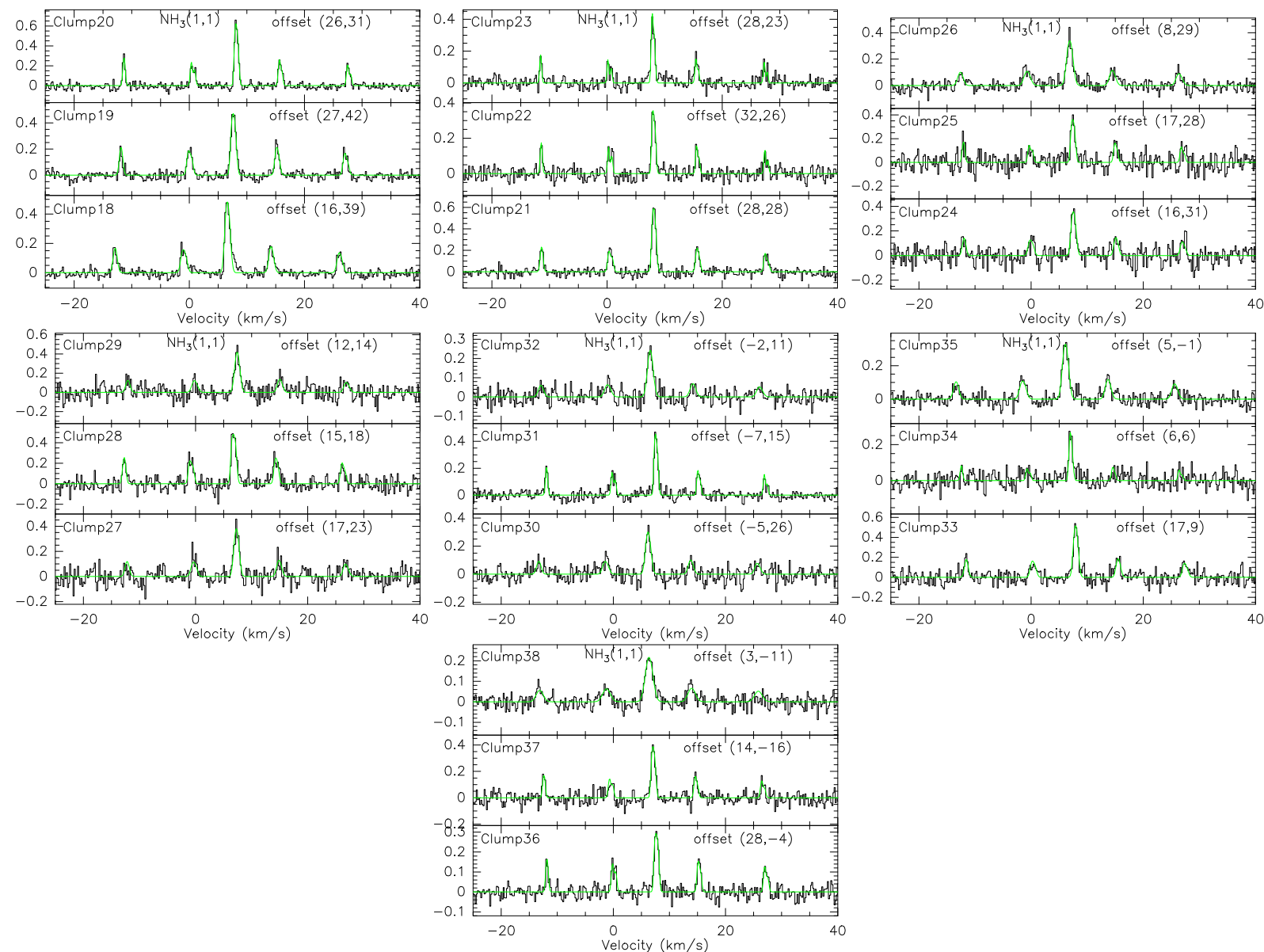

Fig. B.3. $\mathrm{NH}_{3}(1,1)$ spectra towards clumps 18 to 38 . Green color indicates the $\mathrm{NH}_{3}(1,1)$ fitting of the $\mathrm{NH}_{3}(1,1)$ lines. The central position of this area is at $(l, b)=\left(28.59^{\circ}, 3.55^{\circ}\right)$. Offsets in Galactic coordinates (unit: arcmin) are shown in the top right corner of each $\mathrm{NH}_{3}(1,1)$ panel.

Table B.1. Observed parameters of the $\mathrm{NH}_{3}(1,1)$ emission lines detected in the 38 identified clumps (see Sect. 3.2).

\begin{tabular}{cccccccc}
\hline \hline Clump & $\begin{array}{c}\text { Offset } \\
\left({ }^{\prime}{ }^{\prime}\right)\end{array}$ & $\begin{array}{c}\int T_{\mathrm{MB}} \mathrm{d} v \\
\left(\mathrm{~K} \mathrm{~km} \mathrm{~s}^{-1}\right)\end{array}$ & $\begin{array}{c}V_{\mathrm{LSR}} \\
\left(\mathrm{km} \mathrm{s}^{-1}\right)\end{array}$ & $\begin{array}{c}\Delta v \\
\left(\mathrm{~km} \mathrm{~s}^{-1}\right)\end{array}$ & $\begin{array}{c}T_{\mathrm{MB}} \\
(\mathrm{K})\end{array}$ & $\begin{array}{c}\tau \\
T_{\text {ex }} \\
(\mathrm{K})\end{array}$ \\
\hline 01 & $(-17,-41)$ & $0.55(0.11)$ & $6.21(0.02)$ & $0.34(0.05)$ & $0.21(0.03)$ & $1.50(0.83)$ & $3.0(0.1)$ \\
02 & $(1,-19)$ & $0.45(0.03)$ & $7.24(0.03)$ & $1.04(0.07)$ & $0.40(0.04)$ & $0.10(0.14)$ & $\ldots$ \\
03 & $(9,0)$ & $0.61(0.06)$ & $4.98(0.02)$ & $1.01(0.05)$ & $0.41(0.04)$ & $0.68(0.26)$ & $3.7(0.1)$ \\
04 & $(30,-12)$ & $0.88(0.07)$ & $7.19(0.01)$ & $0.71(0.04)$ & $0.48(0.03)$ & $1.15(0.25)$ & $3.4(0.1)$ \\
05 & $(22,-11)$ & $0.26(0.02)$ & $7.04(0.04)$ & $1.22(0.09)$ & $0.24(0.03)$ & $0.10(0.21)$ & $\ldots$ \\
06 & $(17,-8)$ & $0.29(0.03)$ & $6.89(0.05)$ & $1.32(0.14)$ & $0.28(0.05)$ & $0.10(0.20)$ & $\ldots$ \\
07 & $(1,11)$ & $2.15(0.09)$ & $6.71(0.01)$ & $0.65(0.02)$ & $0.83(0.05)$ & $2.29(0.19)$ & $3.7(0.1)$ \\
08 & $(0,14)$ & $2.03(0.11)$ & $7.35(0.01)$ & $0.63(0.03)$ & $0.86(0.07)$ & $1.84(0.19)$ & $3.7(0.1)$ \\
09 & $(4,16)$ & $2.98(0.06)$ & $7.36(0.01)$ & $1.18(0.01)$ & $1.46(0.04)$ & $1.71(0.07)$ & $4.5(0.1)$ \\
10 & $(6,18)$ & $2.86(0.09)$ & $7.46(0.01)$ & $0.81(0.02)$ & $1.02(0.03)$ & $2.66(0.15)$ & $3.8(0.1)$ \\
11 & $(9,22)$ & $2.49(0.09)$ & $7.29(0.01)$ & $0.69(0.02)$ & $1.08(0.03)$ & $1.87(0.14)$ & $4.0(0.1)$ \\
12 & $(12,26)$ & $1.85(0.20)$ & $7.38(0.01)$ & $0.52(0.02)$ & $1.46(0.06)$ & $3.26(0.23)$ & $4.3(0.1)$ \\
13 & $(13,35)$ & $0.86(0.09)$ & $6.84(0.01)$ & $0.57(0.06)$ & $0.38(0.06)$ & $1.47(0.30)$ & $3.2(0.1)$ \\
\hline
\end{tabular}

Notes. The central position is $(l, b)=\left(28.59^{\circ}, 3.55^{\circ}\right)$. Offsets are given in Galactic coordinates. The errors shown in parentheses are fitting uncertainties. In cases where no excitation temperature is given, the $\mathrm{NH}_{3}(1,1)$ line is optically thin and does not allow for a determination of this parameter. 
Table B.1. continued.

\begin{tabular}{|c|c|c|c|c|c|c|c|}
\hline Clump & $\begin{array}{c}\text { Offset } \\
\left({ }^{\prime},{ }^{\prime}\right)\end{array}$ & $\underset{\left(\mathrm{K} \mathrm{km} \mathrm{s}^{-1}\right)}{\int T_{\mathrm{MB}} \mathrm{d} v}$ & $\begin{array}{c}V_{\mathrm{LSR}} \\
\left(\mathrm{km} \mathrm{s}^{-1}\right)\end{array}$ & $\begin{array}{c}\Delta v \\
\left(\mathrm{~km} \mathrm{~s}^{-1}\right)\end{array}$ & $\begin{array}{c}T_{\mathrm{MB}} \\
(\mathrm{K}) \\
\end{array}$ & $\tau$ & $\begin{array}{l}T_{\mathrm{ex}} \\
(\mathrm{K}) \\
\end{array}$ \\
\hline 14 & $(21,34)$ & $1.59(0.09)$ & $7.86(0.01)$ & $0.36(0.01)$ & $0.54(0.05)$ & $2.09(0.25)$ & $3.4(0.1)$ \\
\hline 15 & $(23,37)$ & $0.94(0.09)$ & $7.81(0.02)$ & $0.72(0.06)$ & $0.37(0.06)$ & $2.19(0.36)$ & $3.1(0.1)$ \\
\hline 16 & $(19,41)$ & $1.83(0.09)$ & $7.36(0.01)$ & $0.56(0.02)$ & $0.67(0.03)$ & $2.38(0.20)$ & $3.5(0.1)$ \\
\hline 17 & $(25,45)$ & $2.08(0.08)$ & $7.69(0.01)$ & $0.72(0.02)$ & $0.80(0.05)$ & $2.36(0.16)$ & $3.6(0.1)$ \\
\hline 18 & $(16,39)$ & $0.91(0.05)$ & $6.64(0.01)$ & $0.81(0.03)$ & $0.49(0.06)$ & $1.20(0.16)$ & $3.4(0.1)$ \\
\hline 19 & $(27,42)$ & $1.30(0.09)$ & $7.65(0.01)$ & $0.64(0.03)$ & $0.49(0.03)$ & $2.26(0.26)$ & $3.3(0.1)$ \\
\hline 20 & $(26,31)$ & $1.81(0.09)$ & $8.16(0.01)$ & $0.42(0.01)$ & $0.67(0.05)$ & $2.12(0.25)$ & $3.5(0.1)$ \\
\hline 21 & $(28,28)$ & $1.33(0.09)$ & $8.13(0.01)$ & $0.54(0.02)$ & $0.64(0.03)$ & $1.46(0.24)$ & $3.6(0.1)$ \\
\hline 22 & $(32,26)$ & $1.09(0.11)$ & $8.08(0.01)$ & $0.38(0.03)$ & $0.37(0.03)$ & $2.27(0.47)$ & $3.2(0.1)$ \\
\hline 23 & $(28,23)$ & $1.32(0.13)$ & 7.96(0.01) & $0.33(0.02)$ & $0.39(0.04)$ & $2.47(0.51)$ & $3.2(0.1)$ \\
\hline 24 & $(16,31)$ & $0.81(0.15)$ & $7.56(0.03)$ & $0.68(0.09)$ & $0.39(0.05)$ & $1.69(0.69)$ & $3.2(0.1)$ \\
\hline 25 & $(17,28)$ & $1.05(0.20)$ & $7.46(0.03)$ & $0.52(0.08)$ & $0.39(0.05)$ & $2.33(0.84)$ & $3.2(0.1)$ \\
\hline 26 & $(8,29)$ & $0.53(0.06)$ & $6.91(0.03)$ & $1.11(0.08)$ & $0.36(0.05)$ & $0.83(0.32)$ & $3.4(0.1)$ \\
\hline 27 & $(17,23)$ & $0.64(0.12)$ & $7.29(0.03)$ & $0.83(0.10)$ & $0.39(0.05)$ & $0.89(0.54)$ & $3.4(0.2)$ \\
\hline 28 & $(15,18)$ & $1.81(0.21)$ & $6.79(0.02)$ & $0.59(0.05)$ & $0.53(0.07)$ & $3.39(0.59)$ & $3.3(0.1)$ \\
\hline 29 & $(12,14)$ & $0.67(0.13)$ & $7.43(0.04)$ & $0.89(0.10)$ & $0.42(0.06)$ & $0.83(0.55)$ & $3.5(0.2)$ \\
\hline 30 & $(-5,26)$ & $0.48(0.08)$ & $6.21(0.03)$ & $0.93(0.10)$ & $0.31(0.03)$ & $0.82(0.48)$ & $3.3(0.1)$ \\
\hline 31 & $(-7,15)$ & $1.29(0.10)$ & $7.59(0.01)$ & $0.41(0.02)$ & $0.48(0.04)$ & $2.03(0.35)$ & $3.3(0.1)$ \\
\hline 32 & $(-2,11)$ & $0.29(0.02)$ & $6.59(0.03)$ & $1.08(0.08)$ & $0.25(0.05)$ & $0.10(0.05)$ & \\
\hline 33 & $(17,9)$ & $0.95(0.13)$ & $7.95(0.02)$ & $0.77(0.08)$ & $0.55(0.05)$ & $1.09(0.39)$ & $3.6(0.1)$ \\
\hline 34 & $(6,6)$ & $0.38(0.07)$ & $7.07(0.03)$ & $0.54(0.14)$ & $0.27(0.06)$ & $0.10(0.38)$ & \\
\hline 35 & $(5,-1)$ & $0.56(0.05)$ & $6.13(0.02)$ & $1.05(0.06)$ & $0.34(0.04)$ & $1.04(0.28)$ & $3.3(0.1)$ \\
\hline 36 & $(28,-4)$ & $1.19(0.14)$ & $7.67(0.01)$ & $0.46(0.04)$ & $0.32(0.02)$ & $3.67(0.63)$ & $3.1(0.1)$ \\
\hline 37 & $(14,-16)$ & $1.07(0.13)$ & $7.12(0.02)$ & $0.49(0.04)$ & $0.40(0.03)$ & $2.09(0.54)$ & $3.2(0.1)$ \\
\hline 38 & $(3,-11)$ & $0.28(0.04)$ & $6.37(0.04)$ & $1.47(0.10)$ & $0.22(0.03)$ & $0.42(0.32)$ & $3.4(0.1)$ \\
\hline
\end{tabular}

Table B.2. Observed parameters of the $\mathrm{NH}_{3}(2,2)$ emission lines detected in 17 of the $38 \mathrm{NH}_{3}(1,1)$ clumps (see Sect. 3.2).

\begin{tabular}{cccccc}
\hline \hline Clump & $\begin{array}{c}\text { Offset } \\
\left({ }^{\prime},\right)\end{array}$ & $\begin{array}{c}\int T_{\mathrm{MB}} \mathrm{d} v \\
\left(\mathrm{~K} \mathrm{~km} \mathrm{~s}^{-1}\right)\end{array}$ & $\begin{array}{c}V_{\mathrm{LSR}} \\
\left(\mathrm{km} \mathrm{s}^{-1}\right)\end{array}$ & $\begin{array}{c}\Delta v \\
\left(\mathrm{~km} \mathrm{~s}^{-1}\right)\end{array}$ & $\begin{array}{c}T_{\mathrm{MB}} \\
(\mathrm{K})\end{array}$ \\
\hline 01 & $(-17,-41)$ & $0.07(0.02)$ & $6.19(0.15)$ & $1.17(0.29)$ & $0.06(0.03)$ \\
02 & $(1,-19)$ & $0.17(0.02)$ & $7.21(0.07)$ & $0.99(0.14)$ & $0.16(0.04)$ \\
03 & $(9,0)$ & $0.26(0.02)$ & $5.024(0.05)$ & $1.23(0.11)$ & $0.20(0.04)$ \\
04 & $(30,-12)$ & $0.07(0.01)$ & $7.15(0.04)$ & $0.49(0.10)$ & $0.13(0.04)$ \\
05 & $(22,-11)$ & $0.02(0.01)$ & $10.41(0.08)$ & $0.28(0.12)$ & $0.05(0.01)$ \\
06 & $(17,-8)$ & $0.24(0.03)$ & $6.85(0.07)$ & $1.15(0.19)$ & $0.19(0.04)$ \\
07 & $(1,11)$ & $0.15(0.02)$ & $6.68(0.04)$ & $0.75(0.11)$ & $0.19(0.03)$ \\
08 & $(0,14)$ & $0.19(0.03)$ & $7.13(0.12)$ & $1.82(0.25)$ & $0.10(0.05)$ \\
09 & $(4,16)$ & $0.70(0.03)$ & $7.38(0.03)$ & $1.57(0.07)$ & $0.42(0.05)$ \\
10 & $(6,18)$ & $0.23(0.03)$ & $7.43(0.07)$ & $1.36(0.25)$ & $0.16(0.04)$ \\
11 & $(9,22)$ & $0.13(0.02)$ & $7.25(0.07)$ & $0.99(0.14)$ & $0.13(0.03)$ \\
12 & $(12,26)$ & $0.22(0.03)$ & $7.35(0.05)$ & $0.79(0.13)$ & $0.26(0.04)$ \\
13 & $(13,35)$ & $0.04(0.01)$ & $6.84(0.03)$ & $0.33(0.07)$ & $0.11(0.01)$ \\
14 & $(21,34)$ & $0.15(0.03)$ & $7.26(0.32)$ & $0.23(0.09)$ & $0.04(0.03)$ \\
15 & $(23,37)$ & $0.07(0.01)$ & $7.69(0.08)$ & $0.70(0.16)$ & $0.09(0.04)$ \\
16 & $(19,41)$ & $0.09(0.01)$ & $7.42(0.04)$ & $0.63(0.10)$ & $0.14(0.02)$ \\
17 & $(25,45)$ & $0.15(0.02)$ & $7.54(0.07)$ & $1.21(0.17)$ & $0.12(0.03)$ \\
\hline
\end{tabular}

Notes. The central position is $(l, b)=\left(28.59^{\circ}, 3.55^{\circ}\right)$. Offsets are given in Galactic coordinates. Parameters are derived from Gaussian fits to the spectra. 
Table B.3. Calculated model parameter of $\mathrm{NH}_{3}(1,1)$ and $\mathrm{NH}_{3}(2,2)$ emission lines detected in 17 clumps.

\begin{tabular}{cccccccc}
\hline \hline Clump & $\begin{array}{c}\text { Offset } \\
\left({ }^{\prime}{ }^{\prime}\right)\end{array}$ & $\begin{array}{c}T_{\text {rot }} \\
(\mathrm{K})\end{array}$ & $\begin{array}{c}T_{\text {kin }} \\
(\mathrm{K})\end{array}$ & $\begin{array}{c}N(1,1) \\
\left(\mathrm{cm}^{-2}\right)\end{array}$ & $\begin{array}{c}\text { total- } N\left(\mathrm{NH}_{3}\right)^{(a)} \\
\left(\mathrm{cm}^{-2}\right)\end{array}$ & $\begin{array}{c}N\left(\mathrm{H}_{2}\right)^{(b)} \\
\left(\mathrm{cm}^{-2}\right)\end{array}$ & $\chi($ total-NH $)$ \\
\hline 01 & $(-17,-41)$ & $13.6 \pm 2.9$ & $14.9 \pm 3.8$ & $0.4 \times 10^{14}$ & $3.8 \times 10^{14}$ & $0.9 \times 10^{22}$ & $4.3 \times 10^{-8}$ \\
02 & $(1,-19)$ & $18.3 \pm 2.4$ & $22.5 \pm 3.9$ & $0.1 \times 10^{14}$ & $0.3 \times 10^{14}$ & $1.4 \times 10^{22}$ & $0.2 \times 10^{-8}$ \\
03 & $(9,0)$ & $19.1 \pm 2.1$ & $23.2 \pm 3.5$ & $0.7 \times 10^{14}$ & $3.6 \times 10^{14}$ & $2.5 \times 10^{22}$ & $1.4 \times 10^{-8}$ \\
04 & $(30,-12)$ & $13.9 \pm 1.6$ & $15.5 \pm 2.1$ & $0.8 \times 10^{14}$ & $6.5 \times 10^{14}$ & $2.1 \times 10^{22}$ & $3.1 \times 10^{-8}$ \\
05 & $(22,-11)$ & $14.8 \pm 1.2$ & $16.8 \pm 1.7$ & $0.4 \times 10^{13}$ & $0.3 \times 10^{14}$ & $1.8 \times 10^{22}$ & $0.2 \times 10^{-8}$ \\
06 & $(17,-8)$ & $25.2 \pm 4.3$ & $34.9 \pm 9.2$ & $0.5 \times 10^{13}$ & $0.2 \times 10^{14}$ & $1.9 \times 10^{22}$ & $0.1 \times 10^{-8}$ \\
07 & $(1,11)$ & $11.7 \pm 0.6$ & $12.5 \pm 0.8$ & $1.5 \times 10^{14}$ & $1.9 \times 10^{15}$ & $3.2 \times 10^{22}$ & $5.9 \times 10^{-8}$ \\
08 & $(0,14)$ & $10.1 \pm 1.2$ & $10.6 \pm 1.4$ & $1.2 \times 10^{14}$ & $2.3 \times 10^{15}$ & $2.7 \times 10^{22}$ & $8.9 \times 10^{-8}$ \\
09 & $(4,16)$ & $13.3 \pm 0.6$ & $14.6 \pm 0.8$ & $2.6 \times 10^{14}$ & $2.3 \times 10^{15}$ & $6.3 \times 10^{22}$ & $3.7 \times 10^{-8}$ \\
10 & $(6,18)$ & $10.2 \pm 0.7$ & $10.7 \pm 0.8$ & $2.3 \times 10^{14}$ & $4.3 \times 10^{15}$ & $5.2 \times 10^{22}$ & $8.3 \times 10^{-8}$ \\
11 & $(9,22)$ & $9.9 \pm 0.6$ & $10.5 \pm 0.7$ & $1.5 \times 10^{14}$ & $2.9 \times 10^{15}$ & $2.9 \times 10^{22}$ & $9.9 \times 10^{-8}$ \\
12 & $(12,26)$ & $10.1 \pm 0.4$ & $10.6 \pm 0.5$ & $1.9 \times 10^{14}$ & $3.8 \times 10^{15}$ & $3.6 \times 10^{22}$ & $1.1 \times 10^{-7}$ \\
13 & $(13,35)$ & $13.8 \pm 0.9$ & $15.2 \pm 1.3$ & $0.8 \times 10^{14}$ & $6.4 \times 10^{14}$ & $1.6 \times 10^{22}$ & $4.1 \times 10^{-8}$ \\
14 & $(21,34)$ & $8.6 \pm 1.6$ & $8.9 \pm 1.9$ & $0.7 \times 10^{14}$ & $2.5 \times 10^{15}$ & $1.2 \times 10^{22}$ & $2.1 \times 10^{-7}$ \\
15 & $(23,37)$ & $12.1 \pm 1.9$ & $13.0 \pm 2.5$ & $1.4 \times 10^{14}$ & $1.6 \times 10^{15}$ & $1.3 \times 10^{22}$ & $1.2 \times 10^{-7}$ \\
16 & $(19,41)$ & $11.2 \pm 0.4$ & $11.9 \pm 0.5$ & $1.3 \times 10^{14}$ & $1.8 \times 10^{15}$ & $2.1 \times 10^{22}$ & $8.5 \times 10^{-8}$ \\
17 & $(25,45)$ & $10.2 \pm 0.7$ & $10.7 \pm 0.9$ & $1.7 \times 10^{14}$ & $3.2 \times 10^{15}$ & $2.7 \times 10^{22}$ & $1.2 \times 10^{-7}$ \\
\hline
\end{tabular}

Notes. ${ }^{(a)}$ Total(para+ortho) column densities of $\mathrm{NH}_{3}$, see Sect. 3.4. ${ }^{(b)} \mathrm{H}_{2}$ column densities are taken from Bontemps et al. (2010) and Könyves et al. (2015). The central position is $(l, b)=\left(28.59^{\circ}, 3.55^{\circ}\right)$. Offsets are based on Galactic coordinates.

\section{Appendix C: Uncertainty estimation and derivation of the physical parameters}

The uncertainty of $T_{\text {rot }}$ in Eq. (1) is

$$
\begin{aligned}
\Delta T_{\mathrm{rot}}= & \frac{\partial T_{\mathrm{rot}}}{\partial \tau_{m}(1,1)} \Delta \tau_{m}(1,1)+\frac{\partial T_{\mathrm{rot}}}{\partial T_{\mathrm{MB}}(2,2)} \Delta T_{\mathrm{MB}}(2,2) \\
& +\frac{\partial T_{\mathrm{rot}}}{\partial T_{\mathrm{MB}}(1,1)} \Delta T_{\mathrm{MB}}(1,1)
\end{aligned}
$$

where $\Delta \tau_{m}(1,1), \Delta T_{\mathrm{MB}}(2,2)$ and $\Delta T_{\mathrm{MB}}(1,1)$ are uncertainties of $\tau_{m}(1,1), T_{\mathrm{MB}}(2,2)$ and $T_{\mathrm{MB}}(1,1)$, respectively.

The error of $T_{\text {kin }}$ in Eq. (2) is

$\Delta T_{\text {kin }}=\frac{\partial T_{\text {kin }}}{\partial T_{\text {rot }}} \Delta T_{\text {rot }}$

Uncertainties of $N_{\text {tot }}$ in Eq. (3) are

$\Delta N_{\text {tot }}=\frac{\partial N_{\text {tot }}}{\partial T_{\text {rot }}} \Delta T_{\text {rot }}+\frac{\partial N_{\text {tot }}}{\partial N(1,1)} \Delta N(1,1)$,

where $\Delta N(1,1)$ is the uncertainty of $N(1,1)$ in Eq. (4).

When $\tau \ll 1, N(1,1)$ is given by

$T_{\mathrm{MB}} \propto T_{\mathrm{ex}} \tau$ and

$N(1,1)=\frac{1.65 \times 10^{14}}{v} \frac{J(J+1)}{K^{2}} \Delta v T_{\mathrm{MB}}$,

The uncertainty of $N(1,1)$ then becomes

$$
\begin{aligned}
\Delta N(1,1) & =\frac{\partial N(1,1)}{\partial \Delta v} \Delta \Delta v+\frac{\partial N(1,1)}{\partial T_{\mathrm{MB}}} \Delta T_{\mathrm{MB}} \\
& =\frac{1.65 \times 10^{14}}{v} \frac{J(J+1)}{K^{2}}\left(T_{\mathrm{MB}} \Delta \Delta v+\Delta v \Delta T_{\mathrm{MB}}\right),
\end{aligned}
$$

where $\Delta \Delta v$ represents the error in the line width $\Delta v$. If $\tau \gtrsim 1$, $T_{\text {ex }}$ is obtained from Eq. (5) as a function of $T_{\mathrm{MB}}$ and $\tau$, then the uncertainty of $N(1,1)$ is defined by

$$
\Delta N(1,1)=\frac{\partial N(1,1)}{\partial \Delta v} \Delta \Delta v+\frac{\partial N(1,1)}{\partial \tau_{\text {tot }}} \Delta \tau_{\text {tot }}+\frac{\partial N(1,1)}{\partial T_{\mathrm{MB}}} \Delta T_{\mathrm{MB}}
$$

Conversion of measured FWHM line widths into velocity dispersion: the intensity of a line with Gaussian distribution at radial velocity $v_{\mathrm{r}}$ is

$$
P\left(v_{\mathrm{r}}\right)=\frac{1}{\sqrt{2 \pi} \sigma} \mathrm{e}^{-\frac{1}{2}\left(\frac{v_{\mathrm{r}}-v_{p}}{\sigma}\right)^{2}},
$$

where $v_{\mathrm{p}}$ is the peak velocity, and $\sigma$ is the velocity dispersion. From the definition of the FWHM, we can infer

$$
\mathrm{e}^{-\frac{1}{2}(\Delta v / 2 \sigma)^{2}}=1 / 2 \Rightarrow \sigma=\Delta v /(2 \sqrt{2 \ln 2})=\Delta v / 2.35
$$

where $\Delta v$ is the FWHM.

Conversion of velocity dispersion into thermal contribution to the observed line width (see Eq. (8)):

$v_{\mathrm{obs}}=\sqrt{2} \sigma$.

\section{Appendix D: Fitted velocity and residuals of the velocity fitting}

We have assumed that the observed region shown in Fig. D.1 is a rigid body and fitted its velocity field in a linear form. To check this assumption, we present the distribution of the fitted velocity (Fig. D.1 left panel) and velocity residuals (Fig. D.1 right panel) between the observed velocity and the fitted velocity. Statistics of the velocity residuals are shown in Fig. D.2. From the right panel 

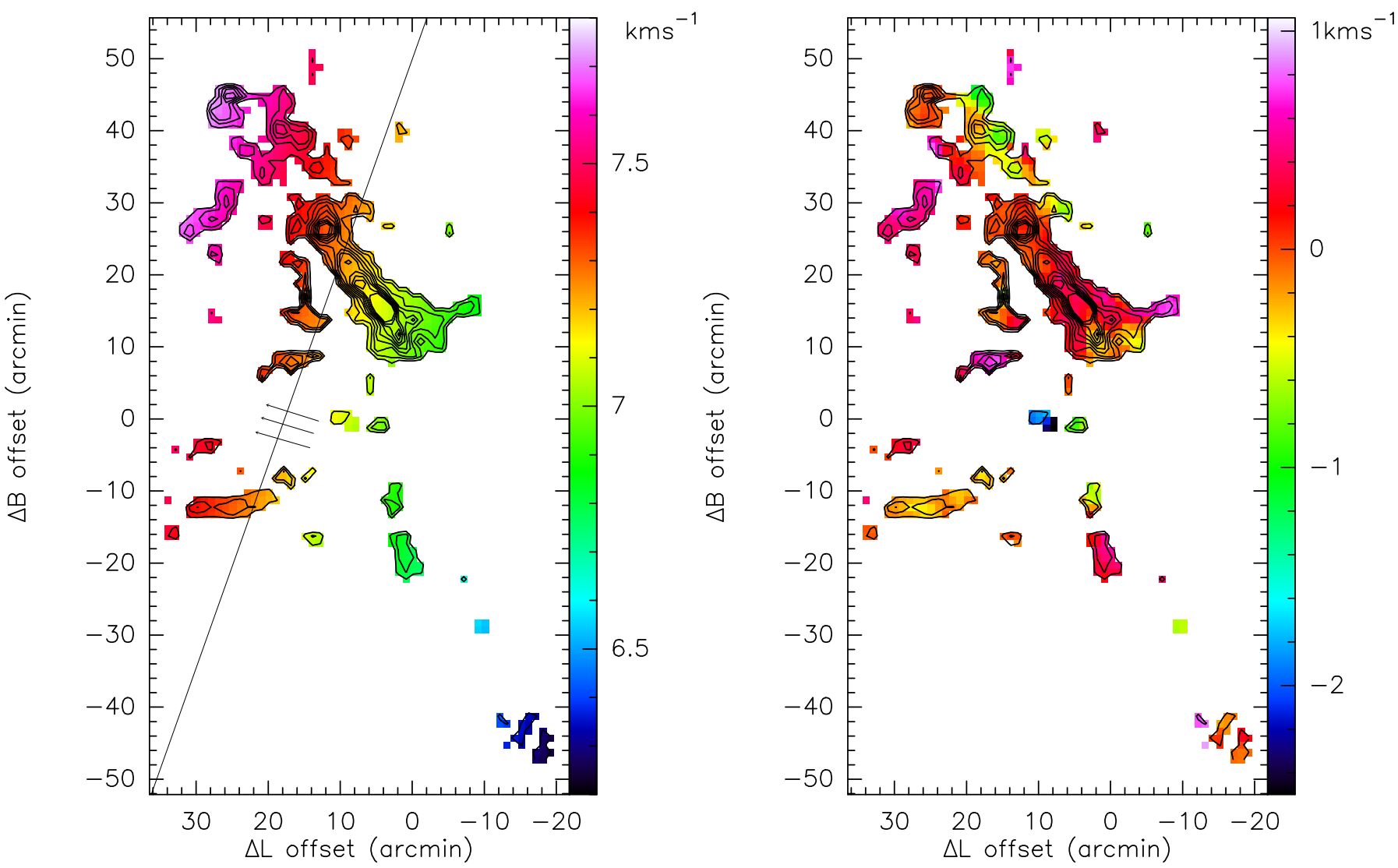

Fig. D.1. Fitted velocity (left $)$ and velocity residual $\left(V_{\text {obs }}-V_{\text {fit }}\right)(r i g h t)$ maps of the $\mathrm{NH}_{3}(1,1)$ lines with $S / N s>5 \sigma$. Contours are as in Figs. 4 and 6. The black solid polyline in the left panel indicates a potential rotation axis, and three black parallel lines perpendicular to the rotation axis show the direction of the velocity gradient.
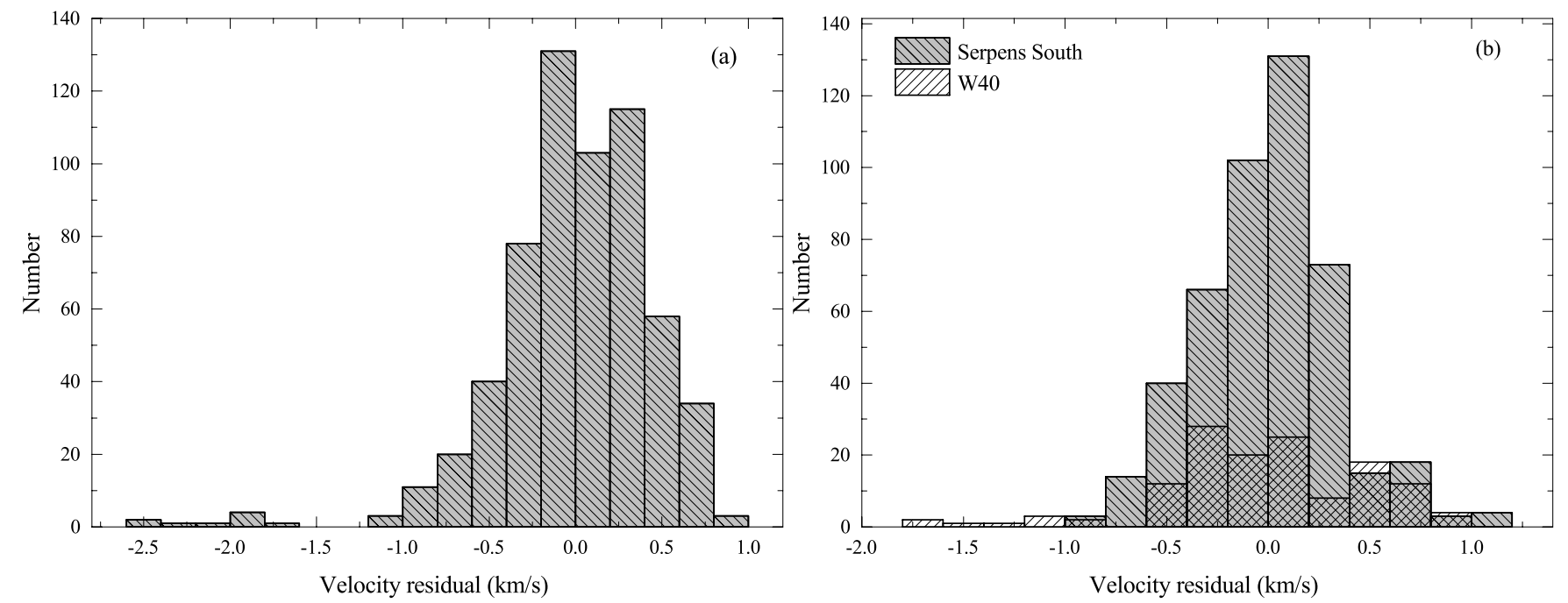

Fig. D.2. Histograms of the velocity residuals $\left(V_{\mathrm{obs}}-V_{\text {fit }}\right)$ derived from our $\mathrm{NH}_{3}(1,1)$ data with $S / N s>5 \sigma:(a)$ entire observed region, $(b)$ Serpens South and W 40.

of Fig. D.1, we can see that most of our velocity residuals are in the range of $V_{\mathrm{obs}}-V_{\mathrm{fit}}=-1$ to $1 \mathrm{~km} \mathrm{~s}^{-1}$, which can be even more clearly seen in Fig. D.2. Specifically, $90 \%$ of the velocity residuals of the shown region, Serpens South alone, and W 40 alone are in the range $V_{\text {obs }}-V_{\text {fit }}<0.65,0.56$, and $0.79 \mathrm{~km} \mathrm{~s}^{-1}$, respectively. Larger velocity residuals are mainly located, in Galactic coordinates, at the western and northeastern part of the Serpens South and the southern part of the W 40. 